\title{
Universiteit
}

Leiden

The Netherlands

\section{The HB40-JUB1 transcriptional regulatory network controls gibberellin homeostasisin Arabidopsis}

Dong, S.; Tarkowska, D.; Sedaghatmehr, M.; Welsch, M.; Gupta, S.; Mueller-Roeber, B.; Balazadeh, S.

\section{Citation}

Dong, S., Tarkowska, D., Sedaghatmehr, M., Welsch, M., Gupta, S., Mueller-Roeber, B., \& Balazadeh, S. (2021). The HB40-JUB1 transcriptional regulatory network controls gibberellin homeostasisin Arabidopsis. Molecular Plant, 15, 1-18. doi:10.1016/j.molp.2021.10.007

Version:

Accepted Manuscript

License:

Creative Commons CC BY 4.0 license

Downloaded from: $\quad$ https://hdl.handle.net/1887/3248580

Note: To cite this publication please use the final published version (if applicable). 


\section{Journal Pre-proof}

The HB40-JUB1 transcriptional regulatory network controls gibberellin homeostasis in Arabidopsis

Shuchao Dong, Danuse Tarkowska, Mastoureh Sedaghatmehr, Maryna Welsch, Saurabh Gupta, Bernd Mueller-Roeber, Salma Balazadeh

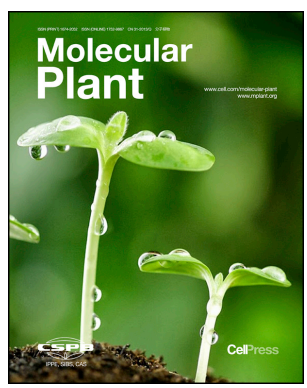

PII: S1674-2052(21)00431-7

DOI: https://doi.org/10.1016/j.molp.2021.10.007

Reference: MOLP 1264

To appear in: MOLECULAR PLANT Accepted Date: 27 October 2021

Please cite this article as: Dong S., Tarkowska D., Sedaghatmehr M., Welsch M., Gupta S., MuellerRoeber B., and Balazadeh S. (2021). The HB40-JUB1 transcriptional regulatory network controls gibberellin homeostasis in Arabidopsis. Mol. Plant. doi: https://doi.org/10.1016/j.molp.2021.10.007.

This is a PDF file of an article that has undergone enhancements after acceptance, such as the addition of a cover page and metadata, and formatting for readability, but it is not yet the definitive version of record. This version will undergo additional copyediting, typesetting and review before it is published in its final form, but we are providing this version to give early visibility of the article. Please note that, during the production process, errors may be discovered which could affect the content, and all legal disclaimers that apply to the journal pertain. 
The HB40-JUB1 transcriptional regulatory network controls gibberellin homeostasis in Arabidopsis

Shuchao Dong ${ }^{1,2}$, Danuse Tarkowska ${ }^{3}$, Mastoureh Sedaghatmehr ${ }^{1}$, Maryna Welsch ${ }^{1,4}$, Saurabh Gupta $^{1,4}$, Bernd Mueller-Roeber ${ }^{1,4}$ and Salma Balazadeh ${ }^{1,2, \S}$

${ }^{1}$ Max-Planck Institute of Molecular Plant Physiology, Am Mühlenberg 1, 14476 PotsdamGolm, Germany.

${ }^{2}$ Institute of Biology, Leiden University, Sylviusweg 72, 2333 BE, Leiden, The Netherlands.

${ }^{3}$ Laboratory of Growth Regulators, Czech Academy of Sciences, Institute of Experimental Botany \& Palacký University, Šlechtitelů 27, CZ-78371 Olomouc, Czech Republic.

${ }^{4}$ University of Potsdam, Institute of Biochemistry and Biology, Karl-Liebknecht-Straße 24-25, Haus 20, 14476 Potsdam-Golm, Germany.

§Corresponding author: Salma Balazadeh; s.balazadeh@biology.leidenuniv.nl

Running title: Homeostatic control of gibberellins by transcription factor HB40

Keywords: Arabidopsis, growth, gibberellin, homeostasis, transcription factor, HB40, JUB1, GA 2-oxidase, DELLA proteins

\section{ORCID IDs:}

Shuchao Dong: 0000-0002-3713-4139

Danuse Tarkowska: 0000-0003-1478-1904

Mastoureh Sedaghatmehr: 0000-0003-0816-5128

Maryna Welsch: 0000-0001-8347-4823

Saurabh Gupta: 0000-0002-5984-852X

Bernd Mueller-Roeber: 0000-0002-1410-464X

Salma Balazadeh: 0000-0002-5789-4071

\section{Email addresses:}

S.D.: sdong@mpimp-golm.mpg.de

D.T.: tarkowska@ueb.cas.cz

M.S.: sedaghatmehr@mpimp-golm.mpg.de

M.W.: molochko@mpimp-golm.mpg.de

S.G.: $\quad$ gupta@mpimp-golm.mpg.de

B.M.-R.: bmr@uni-potsdam.de

S.B.: s.balazadeh@biology.leidenuniv.nl 


\section{SHORT SUMMARY}

Gibberellins (GAs) are plant hormones essential for plant growth and development. In this study, we identified homeobox transcription factor HB40 of the HD-Zip family as a control element of GA homeostasis in Arabidopsis thaliana. HB40 orchestrates a regulatory cascade that involves transcriptional activation of $J U B 1$, a key transcription factor suppressing GA3ox genes and thus GA biosynthesis, and transcriptional activation of GA2ox genes involved in GA inactivation.

\section{ABSTRACT}

The phytohormones gibberellins (GAs) play fundamental roles in almost every aspect of plant growth and development. Although there is good knowledge about GA biosynthetic and signaling pathways, factors contributing to the mechanisms homeostatically controlling GA levels remain largely unclear. Here, we demonstrate that homeobox transcription factor HB40 of the HD-Zip family in Arabidopsis thaliana regulates GA content at two additive control levels. We show that $H B 40$ expression is induced by GA and in turn reduces the levels of endogenous bioactive GAs by a simultaneous reduction of GA biosynthesis and increased GA deactivation. Hence, $H B 40$ overexpression leads to typical GA-deficiency traits, such as small rosettes, reduced plant height, delayed flowering, and male sterility. In contrast, a loss-offunction $h b 40$ mutation enhances GA-controlled growth. Genome-wide RNA-sequencing combined with molecular-genetic analyses revealed that HB40 directly activates transcription of JUNGBRUNNEN1 (JUB1), a key TF repressing growth by suppressing GA biosynthesis and signaling. HB40 also activates genes encoding GA 2-oxidases (GA2oxs) which are major GA catabolic enzymes. The effect of HB40 is ultimately mediated through induction of nuclear growth-repressing DELLA proteins. Our results thus uncover an important role of the HB40/JUB1/GA2ox/DELLA regulatory network in controlling GA homeostasis during plant growth.

\section{INTRODUCTION}

Gibberellins (GAs) are essential plant hormones regulating virtually all aspects of the plant's life including, inter alia, seed germination, hypocotyl and stem elongation, leaf expansion and flower development (Achard and Genschik 2009; Hedden and Sponsel 2015; Binenbaum et al. 2018). Typically, GAs function through the destruction of GRAS domain-containing transcriptional regulators called DELLA proteins. Binding of GA to its receptor GID1 (GIBBERELLIN INSENSITIVE DWARF1) enhances the interaction between GID1 and 
DELLA proteins, leading to the rapid degradation of DELLA proteins via the ubiquitin/proteasome pathway (Sun 2010; Davière and Achard 2013; Thomas et al. 2016). DELLA proteins are master suppressors of plant growth by inhibiting cell proliferation and elongation (Willige et al. 2007), and their function is highly conserved across angiosperms (Gao et al. 2008; Briones-Moreno et al. 2017; Hernández-García et al. 2019). In addition to repressing GA responses, DELLA proteins act as a central node to integrate inputs from light and temperature (Li et al. 2016; Zhou et al. 2017) as well as from other hormone signaling pathways involving brassinosteroids (BRs), auxin, abscisic acid (ABA) and jasmonic acid (JA) (Davière and Achard 2016).

Cellular levels of DELLA proteins are inversely related to levels of bioactive GAs (Achard and Genschik 2009), and lack of functional DELLA results in constitutively activated GA responses, such as elongation growth (Dill and Sun 2001; Ikeda et al. 2001; Itoh et al. 2005; Shahnejat-Bushehri et al. 2016). Thus, the dynamic regulation of endogenous bioactive GA levels throughout the plants' life cycle is essential for optimally balancing growth.

Cellular GA levels are tightly controlled through the regulation of both GA biosynthesis and catabolism. The GA biosynthesis pathway in Arabidopsis thaliana involves multiple enzymes, including GA 20-oxidases (GA20oxs) and GA 33-hydroxylases (GA3oxs), which are 2oxoglutarate-dependent dioxygenase (2ODD) enzymes that catalyze the final steps of bioactive GA synthesis (Mitchum et al. 2006; Sun 2008; Plackett et al. 2012; Martínez-Bello et al. 2015; Hedden 2020). GA20oxs generate $\mathrm{GA}_{9}$ and $\mathrm{GA}_{20}$, which are subsequently converted to biologically active $\mathrm{GAs}\left(\mathrm{GA}_{1}\right.$ and $\left.\mathrm{GA}_{4}\right)$ by $\mathrm{GA} 3$ ox enzymes catalyzing the final and ratelimiting step in GA biosynthesis. GA catabolism is largely dependent on deactivating $2 \beta$ hydroxylation of bioactive GAs and their precursors, mediated by GA 2-oxidases (GA2oxs). The Arabidopsis genome encodes five $\mathrm{C}_{19}$-GA 2oxs (GA2ox 1, 2, 3, 4, and 6) involved in depleting pools of bioactive GAs and their immediate precursors (Rieu et al. 2008; MartínezBello et al. 2015; Takehara et al. 2020).

GA metabolic pathways have been studied extensively and mutants with gains or losses of genes involved in GA biosynthesis or catabolism, leading to changes in GA levels, have been identified in diverse plant species (Yaxley et al. 2001; Magome et al. 2004; Sakamoto et al. 2004; Hu et al. 2008; Plackett et al. 2012; He et al. 2019). However, the molecular mechanisms controlling GA levels remain elusive. This may in part be due to the fact that GA biosynthesis and catabolism (and hence the associated physiological responses) are subject to regulatory networks, involving numerous cues and multiple positive and negative transcriptional feedback 
and feedforward mechanisms (Zentella et al., 2007; Middleton et al. 2012; Fukazawa et al. 2014; Zhang et al. 2019; Wang et al., 2021). Identification and manipulation of the regulatory hubs, for example, transcription factors, that control multiple enzymatic steps of GA metabolic pathways could improve molecular-level understanding of GA homeostasis. For instance, the MADS-box TF OsMADS57 regulates expression of the GA catabolic OsGA2ox3 gene in rice, and a knockdown mutant of OsMADS57 accumulates lower than wild-type (WT) levels of bioactive GAs (Chu et al. 2019). Similarly, a knockdown mutant of the rice HD-Zip class II transcription factor SGD2 (SMALL GRAIN AND DWARF2) reportedly has dramatically reduced $\mathrm{GA}_{1}$ content (Chen et al. 2019). However, direct in planta target genes of SDG2 are unknown. LONG1, a pea orthologue of Arabidopsis bZIP transcription factor HY5 (ELONGATED HYPOCOTYL 5), suppresses GA accumulation by directly promoting GA2ox2 expression (Weller et al. 2009). These examples provide interesting insights, but few TFs that directly affect GA metabolism have been identified, highlighting needs to elucidate more of the transcriptional regulatory networks.

Recently, we demonstrated that JUNGBRUNNEN1 (JUB1), a member of the Arabidopsis NAC (for NAM, ATAF1, 2, and CUC2) TF family, acts as an important negative regulator of GA biosynthesis and signaling by directly transcriptionally repressing the GA biosynthesis gene GA3oxl and activating the transcription of the two DELLA genes GAI and RGLI in Arabidopsis (Shahnejat-Bushehri et al. 2016). However, it remained unclear how JUB1 is integrated into the wider regulatory network that controls GA homeostasis. Here, we report an important extension of the JUB1 control module, which leads to fundamentally new insights into the regulatory networks governing GA homeostasis and GA-mediated growth-regulating networks. We demonstrate that HOMEOBOX PROTEIN 40 (HB40), a TF of the homeodomain-leucine zipper class I (HD-Zip-I) family (Harris et al., 2011), regulates GA levels by decreasing the content of bioactive GAs and increasing the levels of bio-inactive GAs. HB40 has previously been reported to repress shoot branching by promoting abscisic acid (ABA) accumulation, downstream of BRANCHED1 (BRC1), a regulator of shoot branching (Gonzalez-Grandio et al., 2017). The GA homeostasis function of HB40 reported here is mediated by a GA-stimulated enhancement of $H B 40$ expression. This in turn leads to a direct transcriptional activation by HB40 of GA-inactivating genes of the GA2ox family (GA2ox2 and GA2ox6). HB40 also activates the transcription of JUB1 which inhibits the core GA biosynthesis gene GA3oxl. This creates an autoregulatory feedback loop that participates in the control of the activity of $H B 40$. Accordingly, constitutive overexpression of $H B 40$ resulted in reduced cell elongation, smaller rosettes, dwarfism, delayed flowering, and male sterility. In 
contrast, a loss of HB40 function promoted plant growth. Genetic analysis revealed that HB40mediated suppression of growth occurs in a DELLA-dependent manner and requires both, functional JUB1 and GA2ox activities. In summary, our study provides novel insights into the regulatory complexity of the transcriptional control of GA metabolism in plants and suggests new entry points for fine-tuning growth characteristics in crops.

\section{RESULTS}

\section{HB40 Inhibits Growth and Development}

HB40 (AT4G36740) is a member of HD-Zip-I TFs whose expression is rapidly induced by growth-promoting plant hormones GA or brassinosteroid (BR). As shown in Supplemental Figure 1 (A-C), quantitative real-time PCR (qRT-PCR) showed induced expression of $H B 40$ in wild-type (WT) seedlings after $\mathrm{GA}\left(\mathrm{GA}_{3}\right.$ and $\left.\mathrm{GA}_{4}\right)$ or brassinolide (BL) treatment; in contrast its expression was suppressed by GA biosynthesis inhibitor paclobutrazol (Supplemental Figure 1D). To investigate if HB40 plays a role in regulating GA- and/or BR-mediated growth and development, we analyzed Arabidopsis plants overexpressing HB40 fused to a green fluorescent protein (GFP) (hereafter, HB40OX and HB40OX.1 representing two independent transgenic lines) (Supplemental Figure 2A and 2B) and a null mutant of HB40 (hb40-1) (Supplemental Figure 2C-2E).

HB40OX lines produced more compact rosettes than WT counterparts, with smaller leaves and remarkably shorter petioles (nearly absent), while $h b 40-1$ mutants developed significantly larger than WT rosette areas, leaves and petioles (Figure 1A-E and Supplemental Figure 3A -3G). Following this observation, leaf epidermal cells were significantly larger in $h b 40-1$ than WT, but smaller in $H B 40 O X$ (Supplemental Figure 3D and 3E). Introduction of a HB40:HB40-GFP construct into the $h b 40-1$ mutant background restored the growth phenotype of the hb40-1 mutant, confirming that the phenotypes resulted from a loss of HB40 function

\section{(Supplemental Figure 4).}

Furthermore, $h b 40-1$ bolted slightly earlier than WT, while bolting in HB40OX was significantly delayed (Figure 1F). Moreover, HB40OX plants were stunted in height (Figure 1G) and had reduced male fertility, in part due to impaired stamen filament elongation (Figure 1H). We also checked hypocotyl elongation in darkness. HB4OOX hypocotyls were significantly shorter (Figure 1I, and Supplemental Figure 3H and 3I), whereas $h b 40-1$ and WT plants had similar hypocotyl lengths, suggesting an overlapping function between HB40 and other regulators of hypocotyl elongation. Considering the known functional redundancy between $H B 40$ and the two related HD-ZIP-encoding genes $H B 53$ and $H B 21$ in repressing shoot branching (Gonzalez-Grandio et al., 2017), we tested the hypocotyl length of the $h b 40 h b 53$ 
hb21 triple mutant. As shown in Supplemental Figure 3J and 3K, the triple mutant showed significantly longer hypocotyls than WT indicating an overlap of the function of these three TFs in regulating hypocotyl elongation. Taken together, our results demonstrate that HB40 inhibits growth and cell elongation.

\section{HB40 Directly and Positively Regulates JUB1}

To identify putative HB40 targets we first used RNA-seq and compared the transcriptomes of plants expressing HB40 protein (fused to hemagglutinin/HA tag) from an estradiol (Est)inducible promoter (hereafter, HB40-HA-IOE; 2 h Est treatment) (Gonzalez-Grandio et al. 2017) with those of mock-treated controls. We also compared the transcriptomes of $H B 40 O X$ and WT plants. Genes that significantly changed their expression due to $H B 40$ overexpression by at least 2-fold (up or down) were considered further (Supplemental Table 1A and 1B).

We then scored the promoters of all genes for binding by HB40 as determined by DNA affinity purification sequencing (DAP-seq) experiments (O'Malley et al. 2016) and retained all genes containing an HD-Zip binding site within the binding peak (Supplemental Table 1C and 1D). Finally, by comparing the latter two datasets, we identified ten HB40-bound genes commonly affected by $H B 40$ overexpression (eight up- and two downregulated; Figure 2A, Supplemental Table 1E) revealing them as likely direct HB40 targets.

Among those, JUB1 attracted our attention for its recently discovered role as a central transcriptional regulator of GA- and brassinosteroid (BR)-mediated growth (ShahnejatBushehri et al. 2016). In addition, JUB1 was the only transcription factor in the identified set of HB40 target genes (Supplemental Table 1E). Phenotypically, HB40OX and JUB1OX plants strongly resemble each other, suggesting that both transcription factors act in a related molecular network. Thus, to substantiate the model that HB40 regulates JUB 1 transcription, we tested its expression by qRT-PCR in $H B 40$ transgenic lines and observed a significant downregulation of JUB1 transcript abundance in $h b 40-1$, but an upregulation in $H B 40 O X$ compared to WT (Figure 2B). We next studied the expression of JUB1 at different time points $(2,4,6$, and $8 \mathrm{~h})$ after estradiol $(10 \mu \mathrm{M})$ treatment of $H B 40-H A-I O E$ and compared it with data from a mock treatment (no estradiol). Interestingly, expression of JUBl was rapidly (already within $2 \mathrm{~h}$ after estradiol treatment) and significantly upregulated in HB40-HA-IOE plants (Figure 2C), signaling it as an early-responsive target of HB40. In accordance with this, chromatin-immunoprecipitation - quantitative PCR (ChIP-qPCR) confirmed in planta binding of HA-tagged HB40 (HB40-HA) transcription factor to the JUB1 promoter region containing an almost perfect HD-Zip I binding motif (CAATAAATG; 593 bp upstream the translation start site) already one hour after $H B 40$ induction by estradiol, supporting the model that JUBI 
is a bona fide direct target of HB40 (Figure 2D). This conclusion is supported by results from ChIP-qPCR assays that detected significant binding of HB40-GFP to the JUB1 promoter containing the HD-Zip I binding motif in HB40OX plants (Figure 2E). Moreover, His-tagged HB40 protein physically interacts with an infrared dye (IRD)-labeled 40-bp JUB1 promoter fragment containing the HD-Zip I binding motif in an electrophoretic mobility shift assay (EMSA; Figure 2F; the retarded band). There was significant reduction in the intensity of the retarded band upon co-incubation with a competitor (unlabeled promoter fragment), supporting the conclusion of specific binding; mutation of the HD-Zip binding site in the unlabeled competitor diminished its competitive efficiency (Figure 2F). These data clearly demonstrate that HB40 binds to the JUB1 promoter. We also tested activation of the JUB1 promoter by HB40 in transactivation and yeast-one-hybrid (Y1H) assays. As shown in Figure 2G, HB40 activated the JUBI promoter, as revealed by enhanced activity of the luciferase reporter, in mesophyll cell protoplasts of Arabidopsis WT leaves. Y1H demonstrated binding of HB40 to the $J U B 1$ promoter fragment containing the HD-Zip binding site leading to growth of yeast on selective medium (Figure 2H). In conclusion, our results show that HB40 positively and directly regulates $J U B 1$ transcription.

\section{HB40 Requires JUB1 for Growth Suppression}

To determine whether the reduced growth of $H B 40 O X$ plants is due to the regulation of $J U B 1$, and gain insight into the molecular mechanisms through which HB40 modulates growth, we generated double mutant lines overexpressing $H B 40$ in the jubl-1 knockdown mutant (hereafter, HB40OX/jub1-1). Four lines (HB40OX/jub1-1 \#1, \#2, \#9, \#11) with elevated HB40 transcript levels (similar to $H B 40 O X$ plants) and reduced levels of JUB1 transcript were selected for further analysis (Supplemental Figure 5A). We found that the jubl-1 knockdown mutation largely rescues the phenotypes of HB40-overexpressing plants from their signature defects of shorter hypocotyls, smaller rosettes, dwarfism, and delayed flowering (Figure 2I-L and Supplemental Figure 5B-5F) without changing the low $J U B 1$ expression of the mutant background. This result clearly demonstrates that HB40 requires functional JUB1 for growth control. Jubl-1 plants reportedly have significantly larger rosettes and longer hypocotyls than WT plants (Shahnejat-Bushehri et al. 2016), while hypocotyls of $H B 40 O X / j u b 1-1$ were similar to WT (Figure 2I). Likewise, sizes of $H B 40 O X / j u b 1-1$ rosettes were similar to WT, but smaller than jub1-1 rosettes (Figure 2J). These findings highlight the complexity of the gene regulatory network controlled by HB40 and suggest that its functions in growth regulation are not solely mediated through JUB1. 


\section{HB40 Suppresses GA Biosynthesis and Promotes GA Inactivation}

Previous studies revealed that JUB1 directly and negatively regulates both GA and BR biosynthesis genes and that treatment with bioactive $\mathrm{GA}\left(\mathrm{GA}_{4}\right)$ and $\mathrm{BR}$ rescues the short etiolated hypocotyl phenotype of JUBIOX plants (Shahnejat-Bushehri et al. 2016). To assess if HB40 also jointly regulates GA and BR biosynthesis, we first examined the effects of single and combined $\mathrm{GA}_{4}$ and $\mathrm{BL}$ treatments on hypocotyl elongation of light- and dark-grown seedlings. Treatment with BL had no significant effect in the light, and only a weak effect in the dark (even at high concentration, $1 \mu \mathrm{M}$ ) on hypocotyls of $H B 40 O X$ (Figure 3A and Supplemental Figure 6A, C). In contrast, $\mathrm{GA}_{4}$ application significantly increased the length of $H B 40 O X$ hypocotyls in both, light and dark conditions, suggesting that GA deficiency is the main cause of the $H B 40 O X$ short hypocotyl phenotype. Importantly, however, treatment of $H B 40 O X$ plants with $\mathrm{GA}_{4}$ resulted in a partial but not full recovery of hypocotyl growth $(80 \%$ in light, $87 \%$ in darkness), even at a high concentration of $\mathrm{GA}_{4}(1 \mu \mathrm{M})$ (Figure 3A and Supplemental Figure 6B). Hypocotyls of WT, $h b 40-1$, and HB40OX/jub1-1 plants responded similarly to $\mathrm{GA}_{4}$ and/or BL in light and dark (Supplemental Figure 6D and 6E).

We also tested the inductive effect of $\mathrm{GA}_{4}$ on flowering and stem growth. Interestingly, $H B 40 O X$ plants were relatively insensitive to $\mathrm{GA}_{4}$ treatment. $\mathrm{GA}_{4}$ accelerated primary inflorescence growth in WT and $h b 40-1$ seedlings, already at $200 \mathrm{nM} \mathrm{GA}_{4}$. HB40OX seedlings showed no response to this $\mathrm{GA}_{4}$ concentration. Treatment with a higher concentration of $\mathrm{GA}_{4}$ $(1 \mu \mathrm{M})$ triggered elongation of $H B 40 O X$ stems, but to a significantly lesser level than in WT and $h b 40-1$ (Figure 3B and 3C). Collectively, these data indicate that the GA-deficient phenotypes of $H B 40 O X$ plants are not solely due to defects in GA biosynthesis.

Next, we quantified levels of bioactive BR, biologically active GAs and their precursors, as well as bio-inactive GAs in $H B 40$ transgenic and WT plants using ultra-high performance liquid chromatography - tandem mass spectrometry (UHPLC-MS/MS). Levels of BL in HB40OX and hb40-1 plants did not significantly differ from WT (Supplemental Figure 7A), suggesting that HB40 does not regulate BR content in the examined developmental stages. BL levels were significantly higher in HB40OX/jub1-1 than WT plants (Supplemental Figure 7A), which can be explained by the associated reduction in JUB1 activity (Shahnejat-Bushehri et al. 2016). Notably, levels of bioactive $\mathrm{C}_{19}-\mathrm{GAs}\left(\mathrm{GA}_{1}\right.$ and $\left.\mathrm{GA}_{4}\right)$ were significantly lower in $H B 40 O X$ than WT plants. Conversely, GA 4 contents were significantly elevated in $h b 40-1$ (Figure 3D). Endogenous levels of bioactive $\mathrm{GA}_{1}$ and $\mathrm{GA}_{4}$ were indistinguishable between $H B 40 O X / j u b 1$ 1 and WT. Previous studies reported significantly higher levels of $\mathrm{GA}_{1}$ and $\mathrm{GA}_{4}$ in $j u b 1-1$ than 
in WT plants of the same age (Shahnejat-Bushehri et al. 2016) suggesting that mutation of $J U B 1$ restored the reduced $\mathrm{GA}_{1}$ and $\mathrm{GA}_{4}$ contents of $H B 40 O X$.

We did not detect $\mathrm{GA}_{9}$ (immediate precursor of $\mathrm{GA}_{4}$ ) in any genotype, and levels of $\mathrm{GA}_{20}\left(\mathrm{GA}_{1}\right.$ precursor) were not different between genotypes (Supplemental Figure 7B-7D). Among $\mathrm{C}_{20}$ GAs, we did not detect $\mathrm{GA}_{12}$ in any genotype, and no significant differences among genotypes in levels of the others were detected (data not shown).

Furthermore, we assessed levels of GAs in HB40-HA-IOE seedlings after 8 hours of estradiol treatment during which $H B 40$ was highly induced (Figure 2C). The estradiol treatment induced significant reductions in levels of bioactive $\mathrm{GAs}\left(\mathrm{GA}_{1}\right.$ and $\left.\mathrm{GA}_{4}\right)$ in $H B 40-H A-I O E$ seedlings (Figure 3E), further confirming that HB40 negatively regulates bioactive $\mathrm{C}_{19}$-GA levels.

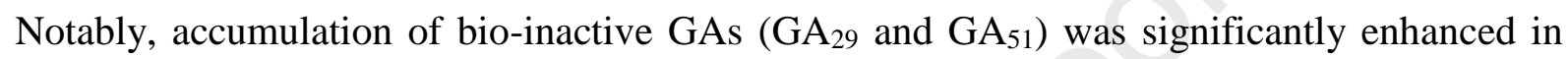
$H B 40 O X$ plants (Figure 3F). This increase of $\mathrm{GA}_{29}$ and $\mathrm{GA}_{51}$ was not observed when $H B 40$ was overexpressed in the jub1-1 background (HB40OX/jubl-1 plants; Figure 3F). This observation can be explained by the fact that more bioactive GAs (GA4 and $\left.\mathrm{GA}_{1}\right)$ are produced due to the missing suppression of GA3oxl and GA3ox2 in those lines due to the lack of JUB1 (Figure 3D; Shahnejat-Bushehri et al. 2016). This likely reduces the availability of $\mathrm{GA}_{51}$ and $\mathrm{GA}_{29}$ precursors for the formation of the bio-inactive GAs. Similar to constitutive overexpressors of $H B 40, H B 40-H A-I O E$ seedlings accumulated higher amounts of bio-inactive GAs $\left(\mathrm{GA}_{29}\right.$ and $\left.\mathrm{GA}_{34}\right)$ under estradiol treatment than under control (mock treatment) conditions (Figure 3G). These results suggest a dual role for HB40 in the regulation of GA biosynthesis and inactivation.

\section{HB40 Directly Regulates the GA Catabolism Genes GA2ox2 and GA2ox6 In Vivo}

To test the hypothesis that accumulation of inactive GAs by HB40 is due to transcriptional regulation of major gibberellin (GA) catabolic enzymes, GA 2-oxidases (GA2oxs), we first measured the expression of all five Arabidopsis $\mathrm{C}_{19}$-GA catabolism genes in HB4O-HA-IOE seedlings. Levels of GA2ox2, GA2ox4 and GA2ox6 transcripts were significantly upregulated upon induction of $H B 40$ (after 8 h of estradiol treatment; Figure 4A). Accordingly, GA2ox2 and GA2ox6 were higher expressed in $H B 40 O X$ than in WT, and lower in $h b 40-1$ (Figure 4B and 4C). The promoters of both genes contain an HD-Zip I binding site (Figure 4D) and EMSA verified physical binding of HB40 to the GA2ox2 and GA2ox6 promoters in vitro (Figure 4E) and ChIP-qPCR confirmed binding of HB40 to both promoters in planta (Figure 4F and 4G). The binding of HB40 to the GA2ox6 but not GA2ox2 promoter was also confirmed in $\mathrm{Y} 1 \mathrm{H}$ assays, and mutation of the HD-Zip I binding site abolished activation of the GA2ox6 promoter 
by HB40 (Figure 4H). Thus, HB40 directly regulates the expression of GA catabolism genes, thereby enhancing GA inactivation via $2 \beta$-hydroxylation.

\section{Inhibition of GA Catabolism Rescues the GA-deficiency Phenotypes of HB40}

To elucidate the biological relevance of the HB40-GA2ox regulation, we overexpressed $H B 40$ in the ga2ox quintuple mutant (hereafter, HB40OX/ga2oxs) and selected lines with enhanced $H B 40$ expression like those of $H B 40 O X$ plants (Supplemental Figure 8A). Interestingly, induction of JUB1 transcription by HB40 was observed in those lines, suggesting that the regulation of JUB1 by HB40 is independent of GA2ox genes (Supplemental Figure 8B). Consistent with previous reports (Rieu et al. 2008) and as shown in Figure 5A-5F, ga2ox quintuple mutants exhibited significantly longer hypocotyls, larger rosette area, longer petioles, accelerated flowering, and an increased plant height compared to WT plants. Mutation of ga2oxs rescued the hypocotyl, rosette and petiole growth deficiency of $H B 40 O X$ to the WT (but not ga2oxs) levels (Figure 5A-5C and Supplemental Figure 8C-8H). HB40OX/ga2ox plants exhibited considerable similarity to ga2oxs counterparts, in terms of flowering time and plant height (Figure 5D-5F). Overall, these results reveal dependency of HB40 on GA2oxs for their growth and development regulation activities.

As already mentioned, $\mathrm{GA}_{4}$ treatment did not fully rescue the short hypocotyls of $H B 40 O X$ and was not effective in induction of flowering in those overexpression plants (Figure 3). To test the hypothesis that the partially insensitive phenotypes of $H B 40 O X$ to GA were in part due to enhanced GA inactivation, we tested the effect of 2,2-dimethyl GA4, a GA 2-oxidase-resistant isoform of $\mathrm{GA}_{4}$ (Yamauchi et al. 2007). The results revealed that treatment with 2,2-dimethyl $\mathrm{GA}_{4}$ fully rescued the short hypocotyls of $H B 40 O X$ (Figure $\mathbf{5 G}$ and $\mathbf{5 H}$ ). With respect to the induction of floral transition and primary inflorescence growth, $0.5 \mu \mathrm{M} \mathrm{GA}_{4}$ and 2,2-dimethyl $\mathrm{GA}_{4}$ were equally effective in WT and $h b 40-1$ seedlings, but only 2,2-dimethyl $\mathrm{GA}_{4}$ was effective in $H B 40 O X$ seedlings (Figure 5I and 5J). These results provide further evidence that HB40 promotes GA catabolism via positive transcriptional regulation of GA2oxs.

\section{HB40 Restrains Growth and Development via DELLAs}

DELLA proteins are key for GA signaling and negative regulators of growth. To further confirm the involvement of HB40 in mitigating GA responses, we determined levels of REPRESSOR OF ga1-3 (RGA) protein, one of the five DELLAs in Arabidopsis, by western blotting. As shown in Figure 6A, RGA levels were significantly higher in $H B 40 O X$ than WT, consistent with the shorter hypocotyls of $H B 40 O X$, while $h b 40-1$ seedlings accumulated less 
RGA than WT. Moreover, in HB40-HA-IOE seedlings, RGA protein accumulated to higher levels upon induction of $H B 40$ by estradiol than in mock-treated samples (Figure 6A). Accordingly, the induction of RGA by HB40 did not occur in $H B 40 O X / j u b 1-1$ and HB40OX/ga2oxs plants (Supplemental Figure 8I). These results suggest that HB40 promotes accumulation of DELLAs by negatively affecting GA levels.

To assess involvement of DELLA proteins in the regulation of growth by HB40, we overexpressed HB40 in the Landsberg erecta (Ler) penta della (gait6, rgat2, 1gl1-1, rgl2-1, rgl3-1) mutant (hereafter, HB40OX/penta della). As control, we overexpressed HB4O in Ler (HB40OX/Ler) (Supplemental Figure 9A). HB40OX/Ler plants showed retarded growth traits, including smaller leaves and rosettes and less elongated inflorescence stems than Ler plants, similar to those observed in HB4OOX plants with Col-0 background. However, the HB40OX/penta della lines exhibited normal growth and maintained an early flowering phenotype, similar to that of the penta della mutant (Figure 6B-6G and Supplemental Figure 9B and 9C). HB40OX/Ler plants had significantly shorter, while the penta della mutant had longer, hypocotyls than Ler plants. The impaired hypocotyl elongation caused by overexpression of $H B 40$ was completely restored by mutations of the DELLA genes (Figure 6H and 6I and Supplemental Figure 9D). We also observed that HB40OX/penta della plants developed normal stamens, unlike $H B 40 O X /$ Ler plants, in which stamen filament elongation is impaired (Figure 6J). These results confirm that the repression of growth and development by HB40 occurs in a DELLA-dependent manner.

\section{DISCUSSION}

The key roles of GA in the regulation of plant growth and development indicate that dynamic modulation of its homeostasis is crucial throughout the entire plants' life cycles. Bioactive GA homeostasis is a tightly regulated process involving both GA biosynthesis and inactivation, and it is under complex feedback control by GA signal transduction pathways (Hedden and Phillips 2000; Sun and Gubler 2004; Zentella et al. 2007; Fukazawa et al. 2017). Diverse endogenous and environmental signals are known to influence the levels of bioactive GAs, partly by modulating the abundance of transcripts of GA biosynthesis and deactivating genes (Yamaguchi and Kamiya 2000; Weller et al. 2009; Son et al. 2010; Shang et al. 2017; Chen et al. 2019). However, only a few TFs regulating GA metabolism by directly controlling the expression of GA metabolizing genes have been identified so far (e.g., Yaish et al. 2010; Gao et al. 2016; Shu et al. 2016; Chu et al. 2019). In this study, we identified and functionally characterized $\mathrm{HB} 40$ as a novel regulator of GA homeostasis, orchestrating both, GA biosynthesis and GA inactivation. Plants overexpressing $H B 4 O$ (HB4OOX) exhibit typical 
growth-related GA-deficiency traits including, inter alia, short hypocotyls, dwarfism, delayed flowering and male sterility. Conversely, loss-of-function mutation of $H B 40(h b 40-1)$ promoted GA-mediated growth.

We revealed that $\mathrm{HB} 40$ directly activates $J U B 1$, a NAC transcription factor suppressing GA biosynthesis (Shahnejat-Bushehri et al. 2016) and genes encoding $\mathrm{C}_{19}$-GA inactivation enzymes (GA 2-oxidases GA2ox2 and GA2ox6) (Figure 2 and 4). In accordance with this, shortly after induction of HB40 (e.g., within $8 \mathrm{~h}$ in HB40-HA-IOE plants) levels of bioactive $\mathrm{C}_{19}$-GAs $\left(\mathrm{GA}_{1}\right.$ and $\left.\mathrm{GA}_{4}\right)$ are strongly downregulated while levels of biologically inactive GAs $\left(\mathrm{GA}_{29}\right.$ and $\left.\mathrm{GA}_{34}\right)$ are significantly upregulated (Figure 3). These results demonstrate a direct link between HB40 levels and contents of bioactive and inactive GAs.

Previous studies have shown that JUB1 regulates GA biosynthesis genes via negative regulation of GA3oxl (directly) and GA3ox2 (indirectly) (Shahnejat-Bushehri et al. 2016). Indeed, among the genes encoding rate-limiting enzymes of GA biosynthesis (GA3oxs and GA20oxs) only GA3ox1 and GA3ox2 were transcriptionally affected by HB40 (significantly downregulated upon induction of HB40 in HB40-IOE seedlings, but upregulated in hb40-1 knockout plants) indicating that HB40 inhibits the synthesis of bioactive GAs mainly by regulating the JUBIGA3oxl,2 circuit (Supplemental Figure 10). Accordingly, induction of GA biosynthesis through knockdown mutation of $J U B 1$ (in jubl-1 plants) significantly restored the phenotypes of $H B 40 O X$ plants, implying that repression of GA biosynthesis through JUB1 is one of the key pathways activated by HB40 (Figure 2). However, neither the knockdown mutation of JUB1, nor exogenous GA treatment completely restored the growth deficiency of HB40overexpressing plants reflecting the importance of catabolic tuning of GA by HB40 and preferential inactivation of bioactive GAs in $H B 40 O X$ plants (Figure $2 \mathbf{I}-2 \mathrm{M}$ and $3 \mathrm{~A}-3 \mathrm{C}$, and

\section{Supplemental Figure 6).}

The Arabidopsis genome contains five $C_{19}-G A 2 o x$ genes $(-1,-2,-3,-4$, and -6$)$ encoding GA inactivation enzymes, all of which confer similar biochemical activities, and are capable of inactivating bioactive $\mathrm{C}_{19}$-GAs $\left(\mathrm{GA}_{1}\right.$ and $\left.\mathrm{GA}_{4}\right)$ and their immediate precursors ( $\mathrm{GA}_{9}$ and $\mathrm{GA}_{20}$ ) (Thomas et al. 1999; Wang et al. 2004; Rieu et al. 2008). GA2ox2 and GA2ox6, the HB40 target genes identified in this study, are the most highly expressed GA2oxs throughout Arabidopsis plants, at all developmental stages (Rieu et al. 2008; Li et al. 2019). However, due to partially overlapping expression patterns and functional redundancy among the GA2ox genes, it had not been possible as yet to assign specific developmental functions to the enzymes they encode (Rieu et al. 2008; Li et al. 2019). The ga2ox quintuple mutant lacking $\mathrm{C}_{19}$-GA 2-oxidase activity 
exhibited significantly longer hypocotyls, larger rosette area, and accelerated flowering. However, overexpression of $H B 40$ in the ga2ox quintuple mutant resulted in traits similar to wild-type (but not ga2ox) plants, especially with regard to hypocotyl elongation and rosette size (Figure 5A-5C and Supplemental Figure 8). This can be explained by high activity of JUB1 in HB40/ga2oxs. As shown in Supplemental Figure 8B, mutation of ga2oxs did not impair induction of JUB1 transcription by HB40. JUB1 expression levels were significantly induced in HB40/ga2oxs, like those in HB40OX, compared to the WT plants. JUB1 expression was not altered in the ga2ox quintuple mutant (Supplemental Figure 8B). Similarly, expression levels of GA2OXs identified as HB40 targets did not change in JUB1OX or jub1-1 transgenic lines (Supplemental Figure 10C), and induction of GA2OXs by HB40 was not compromised in the jubl-1 knockdown mutant (Supplemental Figure 10D and 10E). These data show that the regulation of GA2oxs and JUB1 by HB40 occurs through independent mechanisms. Furthermore, $H B 40 O X$ plants were significantly more responsive to 2,2-dimethyl GA4, a GA analogue resistant to inactivation by GA 2-oxidation, than GA4 (Figure 5G-5J). These results clearly show that both, reduced GA biosynthesis and increased GA inactivation underlie the GA-deficiency phenotypes of $H B 40 O X$ plants. GA-induced degradation of DELLA proteins is a central regulatory mechanism in the GA transduction pathway (Eckardt 2007; Murase et al. 2008). In agreement with the HB40 function in lowering bioactive GA contents, higher accumulation of RGA, an Arabidopsis DELLA protein essential for stem elongation (Dill and Sun 2001; King et al. 2001), was observed in $H B 40$ overexpression lines (both $H B 40 O X$ and HB40-IOE), whereas RGA level was significantly reduced in $h b 40-1$ (Figure 6A). Moreover, the reduced growth triggered by HB40 was fully restored upon overexpression in the genetic background of the penta della mutant. Thus, our results identified an important role of HB40 for the regulation of growth via the DELLA-mediated GA pathway (diagrammed in Figure 7). Interestingly, $H B 40$ is induced by GA treatment. At high GA levels, HB40 activates JUB1 and GA2oxs, leading to a reduction in the abundance of bioactive GAs, thus favoring accumulation of DELLA proteins required for growth suppression. The autoregulatory negative feedback formed by GA and HB40 (Figure 7) adds a new level of complexity to the dynamic model of GA homeostasis. GA levels are controlled by regulatory feedback mechanisms, and DELLA proteins play a central role in the regulation of GA homeostasis (Middleton et al. 2012, Fukazawa et al. 2014; Fukazawa et al. 2017; Tan et al., 2021). We observed that induction of $H B 40$ by $\mathrm{GA}_{4}$ does not occur in the GA-insensitive della mutants RGA $\triangle 17$ and GAI $\triangle 17$ (Feng et al. 2008), revealing that GA-induced expression of $H B 40$ is repressed by DELLAs. Of note, basal expression of $H B 40$ was similar in WT plants and the penta della mutant suggesting 
involvement of other as yet unknown regulatory components (e.g., TFs) (Supplemental Figure 1E and 1F). Alternatively, the unchanged basal expression of $H B 40$ in wild-type and penta della mutants may also be due to the transient nature of the GA effect, an important aspect of hormonal homeostasis. Analyzing this in detail will be an important aspect of future research. An interesting question that remains to be addressed in the future is where (organs) and when (e.g., at which developmental stage and/or upon which environmental condition) does HB40, and potentially other TFs, regulate $J U B 1$ expression. Addressing this in detail requires the analysis of HB40's function in different organ or cell types, and the effect on the expression of $J U B 1$ in those tissues. As seen in Supplemental Figure 11, HB40 shows prominent expression, determined by a promoter-GUS reporter gene fusion, in cotyledon and leaf tips, the shoot apex, primary root tips, and - as previously reported by Gonzalez-Grandio et al. (2017) - in axillary buds. The expression pattern of $H B 40$ thus shows considerable overlap with the expression arrangement of JUB1 in distal leaf areas and primary root tips (Wu et al., 2012). However, we also note that $J U B 1$ has a generally broader expression domain than $H B 40$, which is indicative of additional TFs controlling the expression of JUB1. Discovering those will be an important task in the future.

We previously demonstrated that JUB1, in addition to inhibiting GA biosynthesis, negatively regulates BR biosynthesis. It does so by directly suppressing the gene encoding DWF4, an enzyme catalyzing the rate-limiting step in BR biosynthesis (Wu et al., 2012). However, multiple lines of evidence obtained here indicate that $H B 40 O X$ plants are not pronounced BRdeficient, at least not in the developmental stages we examined (Supplemental Figure 6 and 7). As seen in Supplemental Figure 7A, BR level is significantly elevated in transgenic plants overexpressing HB40 in the jubl-1 knockdown line, demonstrating that HB40 cannot limit BR biosynthesis in the absence of JUB1. This observation fully aligns with our previous report (Shahnejat-Bushehri et al., 2016) where we demonstrated negative regulation of BR level by JUB1. However, in the presence of JUB1, a change in HB40 expression has no obvious effect on BR. A plausible explanation is that JUB1 protein needs to pass a certain threshold level before it can also act on suppressing BR biosynthesis (besides suppressing GA biosynthesis). This threshold level of JUB1 is most likely not reached in $H B 40$ overexpressors. Investigating the threshold model for the action of JUB1 is an interesting task that should be addressed in the future.

Of note, GA metabolism and the signaling of ABA (a central stress-related phytohormone) show extent regulatory interaction to control growth (Davière and Achard, 2013; Golldack et al., 2013; Liu and Hou, 2018). Whether, and to what extent, HB40 modifies this interaction is 
unknown at present. A recent study by González-Grandío et al. (2017) showed that $H B 40$ and the TFs encoded by its homologs HB21 and HB53 regulate the expression of NCED3 which encodes a key enzyme of ABA biosynthesis and, thus, ABA levels in axillary buds of the flower stalk. However, in our study, when analysing non-flowering rosettes, we did not detect significant changes in the expression of ABA biosynthesis and marker genes, including NCED3, when HB40 was modified (Supplemental Table 1 and Supplemental Figure 12). These results suggest that the regulation of ABA biosynthesis by the three HBs occurs in a tissue- and/or developmental stage-specific manner (e.g., in axillary buds).

This study provides illuminating insights into the regulation of GA homeostasis and its impact on plant growth. However, important aspects remain to be resolved. As GA biosynthesis and inactivation genes are differentially expressed between tissues and developmental stages (Yamaguchi et al. 2001; Kaneko et al. 2003; Mitchum et al. 2006; Rieu et al. 2008; Sun 2008; Li et al. 2019) it will be important to precisely map when and where GA biosynthesis and GA inactivation genes are regulated by HB40, and to what extent its regulatory activities overlap. Knowledge of the spatiotemporal regulation of GA metabolism by HB40 (and potentially other TFs) would facilitate the development of practical strategies for modulating GA content in a tissue-specific manner and, hence, optimize plant growth and architecture for enhancing productivity. In addition to this, further research is required to elucidate the environmental and regulatory signals that control $H B 40$ expression. 


\section{METHODS}

\section{Plant Materials and Growth Conditions}

Arabidopsis thaliana ecotypes Col-0 and Ler were used in this study as wild type. Plants were

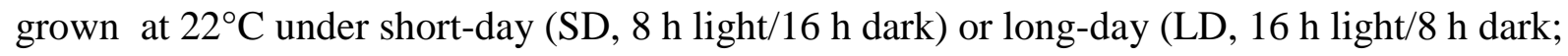
$\left.120 \mu \mathrm{E} \mathrm{m} \mathrm{m}^{-2} \mathrm{~s}^{-1}\right)$ conditions. Surface-sterilized seeds were germinated on half-strength Murashige-Skoog (MS) agar medium containing 1\% sucrose (w/v) and seedlings were grown under $\mathrm{LD}$ condition at $22^{\circ} \mathrm{C}$. Seeds of the penta della mutant, the T-DNA insertion line of $H B 40$ (SALK_115125, renamed as $h b 40-1$ ), and the HB40-IOE line (TRANSPLANTA TPT_4.36740.1C) were obtained from The European Arabidopsis Stock Centre (NASC) seed collection (http://arabidopsis.info/). Seeds of the estradiol-includible line HB40-HA-IOE and the hb21 hb40 hb53-2 triple mutant (Gonzalez-Grandio et al. 2017) and the GA2ox quintuple (ga2oxs) mutant were kindly provided by Dr. Pillar Cubas and Dr. Andy Phillips, respectively. The jub1-1 and JUB1OX lines were described previously (Wu et al. 2012; Shahnejat-Bushehri et al. 2016).

\section{Plasmid Construction and Plant Transformation}

Constructs were generated by Gateway cloning (pENTR Directional TOPO Cloning Kit, Invitrogen; LR Clonas Enzyme Mix, Invitrogen). The CDS of $H B 40$ was amplified from Col0 (WT) cDNA and then cloned into destination vector pK7FWG2.0 (GFP vector; https://gatewayvectors.vib.be) to generate 35S:HB40-GFP. The CaMV $35 S$ promoter was then replaced by the $H B 40$ native promoter $(1,859 \mathrm{bp}$ upstream of the translation start site) using InFusion (Takara) to generate the HB40:HB40-GFP construct. The HB40pro:GUS construct was obtained by cloning the $H B 40$ promoter upstream of the GUS gene in destination vector pKGWFS7.0 (https://gatewayvectors.vib.be). Amplicons generated by PCR were checked for correctness by DNA sequence analysis (Eurofins MWG Operon). Constructs were transformed by floral dip using Agrobacterium tumefaciens strain GV3101. To generate the HB40:HB40GFP/hb40-1 complementation lines, HB40:HB40-GFP was transformed into hb40-1. HB40OX/jub1-1 and HB40OX/ga2oxs plants were generated by transformation of the 35S:HB40:GFP construct into the jub1-1 (Shahnejat-Bushehri et al. 2016) and ga2ox quintuple mutants (Rieu et al. 2008), respectively. To generate the HB40OX/penta della and HB40OX/ga2oxs lines, 35S:HB40:GFP was transformed into penta della and ga2ox quintuple mutants, respectively. For protein expression in Escherichia coli Rosetta, the CDS of $H B 40$ was cloned into destination vector pRMC66-GW to fuse HB40 with the His-tag (Xue, 2005). For transactivation, $1 \mathrm{~kb}$ of the JUB1 promoter containing the $\mathrm{HB} 40$ binding site (CAATAAATG) was cloned into p2GWL7.0 vector harboring the firefly (Photinus pyralis) 
luciferase (FLuc) coding region (Licausi et al. 2011) to generate JUB1:LUC construct. The CDS of HB40 was cloned into pGreen0229-35S (Wu et al. 2012) to generate 35S:HB40. For the yeast-one-hybrid assay, the CDS of HB40 was cloned into pDEST22 (Thermo Fisher Scientific) to generate $H B 40-A D$ construct (HB40 fused with GAL4 activation domain) and a 373-bp fragment of the JUB1 promoter containing the HB40 binding site, a 343-bp fragment of the GA2ox6 promoter containing the HB40 binding site, and a 343-bp fragment of the GA2ox6 promoter with a mutated HB40 binding site were cloned into pTUY1H as described (Ebrahimian-Motlagh et al. 2017). Primers used for cloning are listed in Supplemental Table 2.

\section{Treatments}

To check the effect of phytohormones, Arabidopsis seedlings were grown on half-strength MS agar plates supplemented with synthetic hormones $\mathrm{GA}_{4}$ (Sigma-Aldrich, G7276), brassinolide (Sigma-Aldrich, E1641), or 2,2-dimethyl GA4 (provided by Dr. Peter Hedden). Mock treatments were performed with ethanol (for $\mathrm{GA}_{4}$ treatments; max. $0.01 \%$ [v/v]) or DMSO (for BL treatments, max. $0.0025 \%$ [v/v]). For estradiol (Est) induction, 10-day-old HB40-IOE or HB40-HA-IOE seedlings were transferred to liquid MS medium containing $10 \mu \mathrm{M}$ Est (or $0.1 \%$ [v/v] ethanol as mock treatment) (Wu et al. 2012). The seedlings were kept shaking for 2-8 $\mathrm{h}$ and harvested for further analysis.

\section{RNA Extraction, Sequencing and Data Analysis}

RNA was extracted from 10-day-old seedlings of WT and HB4OOX, and 10-day-old HB40-IOE seedlings treated with or without $10 \mu \mathrm{M}$ estradiol (Est) for six hours. RNA extraction was performed as described previously (Balazadeh et al. 2008; Sedaghatmehr et al. 2016). Library preparation and sequencing were performed at BGI Genomics, China (http://www.bgi.com/). RNA sequencing (RNA-seq) was performed with two (for HB40-IOE seedlings) and three (for WT and HB40OX seedlings) biological replicates per sample on HiSeq4000 (Illumina). The sequencing adaptors and low-quality bases were trimmed using Trimmomatic v0.38 (Bolger et al. 2014), and reads below 25 bp length were discarded. The reads aligning to the ribosomal RNA were filtered out using SortMeRNA (v2.1) (Kopylova et al. 2012). The filtered reads were quantified using kallisto (v0.46.1) (Bray et al. 2016) against the Arabidopsis cDNA sequences obtained from Araport11 (Cheng et al. 2017). Differential expression analysis was carried out using the EdgeR package in R/Bioconductor (Robinson and Oshlack 2010). The $P$-value cutoff $<0.01$ and absolute fold change $\geq 2$ were used to identify differentially expressed genes. The RNA sequencing data are available from the NCBI Bioproject database 
(www.ncbi.nlm.nih.gov/bioproject) under ID PRJNA686245.

\section{Quantitative Real-time PCR}

RNA extraction, cDNA synthesis, and qRT-PCR were performed as described previously (Balazadeh et al. 2008; Sedaghatmehr et al. 2016). Primer sequences are given in Supplemental Table 2. PCR reactions were run on an ABI PRISM 7900HT sequence detection system (Applied Biosystems Applera), and amplification products were visualized using SYBR Green (Life Technologies) Transcripts level of each gene was normalized to ACTIN2 as a reference gene.

\section{Chromatin Immunoprecipitation (ChIP) Assay}

Rosette leaves of HB40OX (with HB40-GFP fusion) and 10-day-old seedlings of HB40-IOE (HB40-HA fusion) treated with or without estradiol were used for ChIP. All experiments were performed according to a published method (Kaufmann et al. 2010). Primers used to amplify $J U B 1, G A 2 o x 2$ and GA2ox6 promoter regions harboring the HB40 binding sites are listed in Supplemental Table 2. Primers annealing to transposable element gene $T A 3$, which lacks an HB40 binding site, were used as negative control. The chromatin extracts were isolated and anti-GFP/anti-HA antibodies (Miltenyi Biotec, Germany) was used to immunoprecipitate protein-DNA complexes (Kaufmann et al. 2010). After reversion of the cross-linking, DNA was purified by QIAquick PCR Purification Kit (Qiagen) and analyzed by qPCR. Col-0 plants or mock-treated seedlings of HB4O-HA-IOE served as negative controls.

\section{DAP-seq Data}

The DAP-seq data were extracted from the experiment published by O'Malley et al. (2016). The computational analysis of the dataset was performed as reported in Zaborowski and Walther (2020) with a promoter region of $1 \mathrm{~kb}$ length, defined as the genomic interval of $-1 \mathrm{bp}$ to $-1,000 \mathrm{bp}$ upstream of the transcription start site (TSS) of a gene.

\section{Electrophoretic Mobility Shift Assay (EMSA)}

Recombinant HB40-His protein was prepared as described previously from E. coli Rosetta (Wu et al. 2012). Protein expression was induced in a 100-mL expression culture using $1 \mathrm{mM}$ IPTG, and cells were harvested $6 \mathrm{~h}$ after induction at $28^{\circ} \mathrm{C}$. $\mathrm{HB} 40$-His protein was isolated from $E$. coli and purified using Protino Ni-IDA Resin (Macherey-Nagel, Düren, Germany). The EMSA experiments were conducted as described previously (Wu et al. 2012). In brief, a 40 bp-long 
fragment of $J U B 1, G A 2 o x 2$ and GA2ox6 promoters containing the HD-Zip class I transcription factor binding motif was selected. DY682-labeled DNA oligos and competitors were obtained from Eurofins (https://www.eurofins.com/). The sequences are listed in Supplemental Table 2. Oligos were annealed by heating to $100^{\circ} \mathrm{C}$, followed by slowly cooling down at room temperature. The binding reaction was performed as described in the Odyssey Infrared EMSA kit instruction manual (LI-COR Biosciences, Lincoln, USA). DNA-protein complexes were separated on $6 \%$ retardation gel, and the DY700 signal was detected using the Odyssey Infrared Imaging System (LI-COR Biosciences).

\section{Transactivation Assay}

Arabidopsis mesophyll cell protoplasts were isolated as described from plants grown under SD condition (Yoo et al. 2007). The construct carrying the 1-kb JUB1 promoter (upstream of the translation start site) in front of the firefly luciferase coding region (JUB1:LUC) was cotransformed in the presence or absence of the 35S:HB40 plasmid. Co-transfected UBQ10:GUS vector was used for the normalization of transformation efficiency (Boudsocq et al. 2010). Twenty $\mu$ g DNA was used for the transient transformation of protoplasts. Sixteen hours after incubation, protoplasts were harvested for reporter assay or kept in $-80^{\circ} \mathrm{C}$ until further analysis. Proteins were extracted by adding $100 \mu \mathrm{L}$ protoplast lysis buffer containing $25 \mathrm{mM}$ Trisphosphate (pH 7.8), 2 mM CDTA, 2 mM DTT, 10\% (v/v) glycerol and 1\% (v/v) Triton X-100. The resulting suspension was briefly vortexed. Firefly luciferase activity was quantified using the Luc-Pair Firefly Luciferase HT Assay Kit (GeneCopoeia). GUS activity was measured by the fluorimetric GUS assay. Data were collected as ratios (firefly luciferase activity/GUS activity).

\section{Yeast-one-hybrid Assay}

The JUB1pro373-pTUY1H (LEU2 selection marker; 373-bp JUB1 promoter driving the expression of imidazole glycerolphosphate dehydratase (HIS3) reporter), GA2ox6pro343pTUY1H (LEU2 selection marker; 343-bp GA2ox6 promoter driving the expression of HIS3 reporter) and GA2ox6pro343-mut-pTUY1H constructs were transformed into yeast strain Y187. The HB40-AD pDEST22 (TRP1 selection marker) construct was transformed into yeast strain YM4271. Examination of the interaction between HB40 and the $373 \mathrm{bp}$ long JUB1 and $343 \mathrm{bp}$ GA2ox6 promoter fragments was done on SD medium lacking the essential amino acids Leu, Trp, and His (-L-T-H) in the absence or presence of different concentrations of 3-amino1,2,4-triazole (3-AT) to exclude false positive interactions. 


\section{Western Blot}

Total protein from plant material was extracted as described (Shahnejat-Bushehri et al. 2016). Protein concentration was measured using the BCA Protein Assay kit (Thermofisher Scientific, 23225). Proteins were separated by sodium dodecyl sulphate-polyacrylamide gel electrophoresis on $12 \%$ polyacrylamide gels. For immunoblot analysis, proteins were blotted onto a Protan nitrocellulose membrane (Sigma-Aldrich, 10401396). Rabbit anti-RGA polyclonal antibody (Agrisera, AS11 1630; 1:1,000) was used. IRDye 800CW-conjugated goat anti-rabbit $\operatorname{IgG}(\mathrm{H}+\mathrm{L})$ antibody was used as a secondary antibody at 1:10,000 dilution (LICOR Biosciences). RbcL detected by Ponceau S staining was used as the loading control.

\section{Phytohormone Analysis}

GAs were analyzed as described with some modifications (Urbanova et al. 2013). Briefly, 30 mg Arabidopsis tissue was ground with $1 \mathrm{~mL}$ of ice-cold $80 \%(\mathrm{v} / \mathrm{v})$ acetonitrile containing 5\% (v/v) formic acid. Samples were then extracted overnight at $4^{\circ} \mathrm{C}$ using a benchtop rotator Stuart SB3 (Bibby Scientific) after adding internal gibberellin standards (OlChemIm, Czech Republic). The homogenates were centrifuged, supernatants purified using mixed-mode SPE cartridges (Waters, Ireland) and analyzed by UHPLC-MS/MS (Micromass, UK). GAs were detected using multiple reaction monitoring modes of the transition of the ion $[\mathrm{M}-\mathrm{H}]^{-}$to the appropriate product ion. The standard isotope dilution method (Rittenberg and Foster 1940) was used to quantify GAs levels, and Masslynx 4.1 software (Waters, USA) was used for data analysis.

\section{Confocal Laser Scanning Microscopy (CLSM)}

Arabidopsis root cells were stained with $10 \mathrm{mg} / \mathrm{L}$ DAPI (4',6-diamidino-2-phenylindole) solution for $30 \mathrm{~min}$ to label the nuclei. The CLSM analysis was performed as described previously to visualize HB40-GFP in nuclei (Sampathkumar and Wightman 2015).

\section{Gene Codes}

Arabidopsis gene codes are: ACTIN2, AT3G18780; HB40, AT4G36740; JUB1, AT2G43000; GA2ox2, AT1G30040; GA2ox6, AT1G02400; TA3, AT1G37110. Additional gene codes are given in Supplemental Table 1.

\section{ACKNOWLEDGEMENTS}

We thank the MPI of Molecular Plant Physiology (MPI-MP) for supporting our research. SD thanks the China Scholarship Council for financial support. The work was further financially supported by the European Regional Development Fund Project 'Centre for Experimental Plant 
Biology' (No. CZ.02.1.01/0.0/0.0/16_019/0000738) and the Czech Science Foundation (Nr. 18-10349S). We thank Dr. Pillar Cubas (Centro Nacional de Biotecnología-CSIC, Madrid, Spain) for providing the estradiol inducible line HB4O-HA-IOE and the $h b 21 \mathrm{hb} 40 \mathrm{hb53}-2$ triple mutant, Dr. Andy Phillips (Rothamsted Research, Harpenden, UK) for providing the ga2ox quintuple mutant, Dr. Peter Hedden (Rothamsted Research) for providing 2,2-dimethyl GA4, and Dr. Marie Boudsocq (Universite Paris-Saclay, France) for providing vectors for transactivation assays. We thank our colleagues from the MPI-MP: Dr. Dirk Walther and his 'Bioinformatics' group for supporting the analysis of DAP-seq data; Karina Schulz for support in yeast one-hybrid assays; and Dr. Karin Köhl and her team for plant care.

\section{AUTHOR CONTRIBUTIONS}

SB and BM-R initiated the study; SB designed the research and supervised the work. SD performed the experiments. DT performed hormone measurements. MS performed western blotting analysis. MW helped with transactivation assays. SG helped with gene expression analysis. SB and SD wrote the manuscript. All authors agreed with the final manuscript and its submission for publication.

\section{COMPETING INTERESTS}

Authors declare no competing financial interests.

\section{Supplemental Figures}

Supplemental Figure 1. GA and BL induce the expression of $H B 40$ and GA-induced expression of $H B 40$ is repressed by DELLAs.

Supplemental Figure 2. Expression of $H B 40$ in $H B 40 O X$ and $h b 40-1$ mutants.

Supplemental Figure 3. HB40 inhibits growth and cell expansion.

Supplemental Figure 4. Growth characteristics of $h b 40-1$ are restored by introduction of HB40:HB40-GFP.

Supplemental Figure 5. Growth and developmental defects of $H B 40 O X$ are largely restored by mutation of $J U B 1$.

Supplemental Figure 6. The response of $H B 40 O X$ to GA and BR treatments.

Supplemental Figure 7. Concentration of brassinolide and direct precursors of bioactive $\mathrm{GA}_{1}$ in $H B 40$ transgenic lines and WT plants.

Supplemental Figure 8. Molecular and phenotypic characterization of HB40OX/ga2oxs lines. 
Supplemental Figure 9. Molecular and phenotypic characterization of HB40OX/penta della lines.

Supplemental Figure 10. Expression level of GA biosynthesis genes and GA2ox2 and GA2ox6 in $H B 40$ and $J U B 1$ transgenic lines.

Supplemental Figure 11. Expression of $H B 40$ determined by promoter-GUS analysis.

Supplemental Figure 12. Expression level of ABA biosynthesis and responsive genes is unchanged between WT and $h b 40-1$.

\section{REFERENCES}

Achard P, Genschik P (2009) Releasing the brakes of plant growth: how GAs shutdown DELLA proteins. Journal of Experimental Botany 60 (4):1085-1092. doi:10.1093/jxb/ern301

Balazadeh S, Riaño-Pachón D, Mueller-Roeber B (2008) Transcription factors regulating leaf senescence in Arabidopsis thaliana. Plant Biology 10:63-75

Binenbaum J, Weinstain R, Shani E (2018) Gibberellin Localization and Transport in Plants. Trends Plant Sci 23 (5):410-421. doi:10.1016/j.tplants.2018.02.005

Boudsocq M, Willmann MR, McCormack M, Lee H, Shan L, He P, Bush J, Cheng SH, Sheen J (2010) Differential innate immune signalling via $\mathrm{Ca}^{2+}$ sensor protein kinases. Nature 464(7287):418-22. doi:10.1038/nature08794.

Bolger AM, Lohse M, Usadel B (2014) Trimmomatic: a flexible trimmer for Illumina sequence data. Bioinformatics 30 (15):2114-2120. doi:10.1093/bioinformatics/btu170

Bray NL, Pimentel H, Melsted P, Pachter L (2016) Near-optimal probabilistic RNA-seq quantification. Nat Biotechnol 34 (5):525-527. doi:10.1038/nbt.3519

Briones-Moreno A, Hernández-García J, Vargas-Chávez C, Romero-Campero FJ, Romero JM, Valverde F, Blázquez MA (2017) Evolutionary Analysis of DELLA-Associated Transcriptional Networks. Front Plant Sci 8:626. doi:10.3389/fpls.2017.00626

Cai S, Lashbrook CC (2008) Stamen abscission zone transcriptome profiling reveals new candidates for abscission control: enhanced retention of floral organs in transgenic plants overexpressing Arabidopsis ZINC FINGER PROTEIN2. Plant Physiology 146 (3):1305-1321

Chen W, Cheng Z, Liu L, Wang M, You X, Wang J, Zhang F, Zhou C, Zhang Z, Zhang H (2019) Small Grain and Dwarf 2, encoding an HD-Zip II family transcription factor, regulates plant development by modulating gibberellin biosynthesis in rice. Plant Science 288:110208

Cheng CY, Krishnakumar V, Chan AP, Thibaud-Nissen F, Schobel S, Town CD (2017) Araport11: a complete reannotation of the Arabidopsis thaliana reference genome. Plant J 89 (4):789-804. doi:10.1111/tpj.13415

Chu Y, Xu N, Wu Q, Yu B, Li X, Chen R, Huang J (2019) Rice transcription factor OsMADS57 regulates plant height by modulating gibberellin catabolism. Rice 12 (1):38

Davière JM, Achard P (2013) Gibberellin signaling in plants. Development 140 (6):1147-1151. doi:10.1242/dev.087650

Davière JM, Achard P (2016) A Pivotal Role of DELLAs in Regulating Multiple Hormone Signals. Mol Plant 9 (1):10-20. doi:10.1016/j.molp.2015.09.011

Della Proteins: Master Regulators of Gibberellin-Responsive Growth and Development (2016). In: Annual Plant Reviews, Volume 49. pp 189-228. doi:https://doi.org/10.1002/9781119210436.ch7

Dill A, Sun T-P (2001) Synergistic derepression of gibberellin signaling by removing RGA and GAI function in Arabidopsis thaliana. Genetics 159 (2):777-785

Ebrahimian-Motlagh S, Ribone PA, Thirumalaikumar VP, Allu AD, Chan RL, Mueller-Roeber B, Balazadeh S (2017) JUNGBRUNNEN1 confers drought tolerance downstream of the HD-Zip I transcription factor AtHB13. Frontiers in Plant Science 8:2118 
Eckardt NA (2007) GA perception and signal transduction: molecular interactions of the GA receptor GID1 with GA and the DELLA protein SLR1 in rice. Am Soc Plant Biol,

Feng S, Martinez C, Gusmaroli G, Wang Y, Zhou J, Wang F, Chen L, Yu L, Iglesias-Pedraz JM, Kircher S, Schäfer E, Fu X, Fan LM, Deng XW. (2008) Coordinated regulation of Arabidopsis thaliana development by light and gibberellins. Nature 451(7177):475-479. doi: 10.1038/nature06448. PMID: 18216856; PMCID: PMC2562044.

Fukazawa J, Mori M, Watanabe S, Miyamoto C, Ito T, Takahashi Y (2017) DELLA-GAF1 complex is a main component in gibberellin feedback regulation of GA20 oxidase 2. Plant Physiology 175 (3): 1395-1406

Fukazawa J, Teramura H, Murakoshi S, Nasuno K, Nishida N, Ito T, Yoshida M, Kamiya Y, Yamaguchi S, Takahashi Y (2014) DELLAs function as coactivators of GAI-ASSOCIATED FACTOR1 in regulation of gibberellin homeostasis and signaling in Arabidopsis. Plant Cell 26 (7):2920-2938. doi:10.1105/tpc.114.125690

Gao S, Fang J, Xu F, Wang W, Chu C (2016) Rice HOX12 Regulates Panicle Exsertion by Directly Modulating the Expression of ELONGATED UPPERMOST INTERNODE1. Plant Cell 28 (3):680-695. doi:10.1105/tpc. 15.01021

Gao XH, Huang XZ, Xiao SL, Fu XD (2008) Evolutionarily conserved DELLA-mediated gibberellin signaling in plants. Journal of Integrative Plant Biology 50 (7):825-834

Golldack D, Li C, Mohan H, Probst N. (2013) Gibberellins and abscisic acid signal crosstalk: living and developing under unfavorable conditions. Plant Cell Rep. 32:1007-1016.

Gonzalez-Grandio E, Pajoro A, Franco-Zorrilla JM, Tarancon C, Immink RG, Cubas P (2017) Abscisic acid signaling is controlled by a BRANCHED1/HD-ZIP I cascade in Arabidopsis axillary buds. Proceedings of the National Academy of Sciences USA 114 (2):E245-e254. doi:10.1073/pnas.1613199114

Harris JC, Hrmova M, Lopato S, Langridge P. (2011) Modulation of plant growth by HD-Zip class I and II transcription factors in response to environmental stimuli. New Phytol. 90(4):823-37. doi: 10.1111/j.1469-8137.2011.03733.x.

He H, Liang G, Lu S, Wang P, Liu T, Ma Z, Zuo C, Sun X, Chen B, Mao J (2019) Genome-wide identification and expression analysis of GA2ox, GA3ox, and GA20ox are related to gibberellin oxidase genes in grape (Vitis vinifera L.). Genes 10 (9):680

Hedden P. The current status of research on gibberellin biosynthesis (2020). Plant Cell Physiol. doi: $10.1093 / \mathrm{pcp} / \mathrm{pcaa} 092$.

Hedden P, Phillips AL (2000) Gibberellin metabolism: new insights revealed by the genes. Trends in Plant Science 5 (12):523-530

Hedden P, Sponsel V (2015) A Century of Gibberellin Research. J Plant Growth Regul 34 (4):740760. doi:10.1007/s00344-015-9546-1

Hernández-García J, Briones-Moreno A, Dumas R, Blázquez MA (2019) Origin of GibberellinDependent Transcriptional Regulation by Molecular Exploitation of a Transactivation Domain in DELLA Proteins. Mol Biol Evol 36 (5):908-918. doi:10.1093/molbev/msz009

Hu J, Mitchum MG, Barnaby N, Ayele BT, Ogawa M, Nam E, Lai W-C, Hanada A, Alonso JM, Ecker JR (2008) Potential sites of bioactive gibberellin production during reproductive growth in Arabidopsis. The Plant Cell 20 (2):320-336

Ikeda A, Ueguchi-Tanaka M, Sonoda Y, Kitano H, Koshioka M, Futsuhara Y, Matsuoka M, Yamaguchi J (2001) slender rice, a constitutive gibberellin response mutant, is caused by a null mutation of the SLRI gene, an ortholog of the height-regulating gene GAI/RGA/RHT/D8. The Plant Cell 13 (5):999-1010

Itoh H, Shimada A, Ueguchi-Tanaka M, Kamiya N, Hasegawa Y, Ashikari M, Matsuoka M (2005) Overexpression of a GRAS protein lacking the DELLA domain confers altered gibberellin responses in rice. The Plant Journal 44 (4):669-679

Kaneko M, Itoh H, Inukai Y, Sakamoto T, Ueguchi-Tanaka M, Ashikari M, Matsuoka M (2003) Where do gibberellin biosynthesis and gibberellin signaling occur in rice plants? The Plant Journal 35 (1):104-115

Kaufmann K, Muino JM, Østerås M, Farinelli L, Krajewski P, Angenent GC (2010) Chromatin immunoprecipitation (ChIP) of plant transcription factors followed by sequencing (ChIPSEQ) or hybridization to whole genome arrays (ChIP-CHIP). Nature Protocols 5 (3):457 
King KE, Moritz T, Harberd NP (2001) Gibberellins are not required for normal stem growth in Arabidopsis thaliana in the absence of GAI and RGA. Genetics 159 (2):767-776

Kopylova E, Noé L, Touzet H (2012) SortMeRNA: fast and accurate filtering of ribosomal RNAs in metatranscriptomic data. Bioinformatics 28 (24):3211-3217. doi:10.1093/bioinformatics/bts611

Li C, Zheng L, Wang X, Hu Z, Zheng Y, Chen Q, Hao X, Xiao X, Wang X, Wang G (2019) Comprehensive expression analysis of Arabidopsis GA2-oxidase genes and their functional insights. Plant Science 285:1-13

Li K, Yu R, Fan LM, Wei N, Chen H, Deng XW (2016) DELLA-mediated PIF degradation contributes to coordination of light and gibberellin signalling in Arabidopsis. Nat Commun 7:11868. doi:10.1038/ncomms 11868

Licausi F, Weits DA, Pant BD, Scheible WR, Geigenberger P, van Dongen JT (2011) Hypoxia responsive gene expression is mediated by various subsets of transcription factors and miRNAs that are determined by the actual oxygen availability. New Phytologist 190 (2):442456

Liu X, Hou X. (2018) Antagonistic Regulation of ABA and GA in metabolism and signaling pathways. Front Plant Sci. 9:251.

Magome H, Yamaguchi S, Hanada A, Kamiya Y, Oda K (2004) dwarf and delayed-flowering 1, a novel Arabidopsis mutant deficient in gibberellin biosynthesis because of overexpression of a putative AP2 transcription factor. The Plant Journal 37 (5):720-729

Martínez-Bello L, Moritz T, López-Díaz I (2015) Silencing C19-GA 2-oxidases induces parthenocarpic development and inhibits lateral branching in tomato plants. Journal of Experimental Botany 66 (19):5897-5910

Middleton AM, Úbeda-Tomás S, Griffiths J, Holman T, Hedden P, Thomas SG, Phillips AL, Holdsworth MJ, Bennett MJ, King JR, Owen MR (2012) Mathematical modeling elucidates the role of transcriptional feedback in gibberellin signaling. Proc Natl Acad Sci U S A 109 (19):7571-7576. doi:10.1073/pnas.1113666109

Mitchum MG, Yamaguchi S, Hanada A, Kuwahara A, Yoshioka Y, Kato T, Tabata S, Kamiya Y, Sun Tp (2006) Distinct and overlapping roles of two gibberellin 3-oxidases in Arabidopsis development. The Plant Journal 45 (5):804-818

Murase K, Hirano Y, Sun T-p, Hakoshima T (2008) Gibberellin-induced DELLA recognition by the gibberellin receptor GID1. Nature 456 (7221):459-463

O’Malley RC, Huang S-sC, Song L, Lewsey MG, Bartlett A, Nery JR, Galli M, Gallavotti A, Ecker JR (2016) Cistrome and epicistrome features shape the regulatory DNA landscape. Cell 165 (5): $1280-1292$

Plackett AR, Powers SJ, Fernandez-Garcia N, Urbanova T, Takebayashi Y, Seo M, Jikumaru Y, Benlloch R, Nilsson O, Ruiz-Rivero O (2012) Analysis of the developmental roles of the Arabidopsis gibberellin 20-oxidases demonstrates that GA20oxl,-2, and -3 are the dominant paralogs. The Plant Cell 24 (3):941-960

Rieu I, Eriksson S, Powers SJ, Gong F, Griffiths J, Woolley L, Benlloch R, Nilsson O, Thomas SG, Hedden P (2008) Genetic analysis reveals that C19-GA 2-oxidation is a major gibberellin inactivation pathway in Arabidopsis. The Plant Cell 20 (9):2420-2436

Rittenberg D, Foster G (1940) A new procedure for quantitative analysis by isotope dilution, with application to the determination of amino acids and fatty acids. Journal of Biological Chemistry 133:737-744

Robinson MD, Oshlack A (2010) A scaling normalization method for differential expression analysis of RNA-seq data. Genome Biol 11 (3):R25. doi:10.1186/gb-2010-11-3-r25

Sakamoto T, Miura K, Itoh H, Tatsumi T, Ueguchi-Tanaka M, Ishiyama K, Kobayashi M, Agrawal GK, Takeda S, Abe K (2004) An overview of gibberellin metabolism enzyme genes and their related mutants in rice. Plant Physiology 134 (4):1642-1653

Sampathkumar A, Wightman R (2015) Live cell imaging of the cytoskeleton and cell wall enzymes in plant cells. Methods Mol Biol 1242:133-141. doi:10.1007/978-1-4939-1902-4_12

Sedaghatmehr M, Mueller-Roeber B, Balazadeh S (2016) The plastid metalloprotease FtsH6 and small heat shock protein HSP21 jointly regulate thermomemory in Arabidopsis. Nature Communications 7 (1):1-14 
Shahnejat-Bushehri S, Tarkowska D, Sakuraba Y, Balazadeh S (2016) Arabidopsis NAC transcription factor JUB1 regulates GA/BR metabolism and signalling. Nature Plants 2 (3):1-9

Shang M, Wang X, Zhang J, Qi X, Ping A, Hou L, Xing G, Li G, Li M (2017) Genetic regulation of GA metabolism during vernalization, floral bud initiation and development in Pak Choi (Brassica rapa ssp. chinensis Makino). Frontiers in Plant Science 8:1533

Shu K, Chen Q, Wu Y, Liu R, Zhang H, Wang P, Li Y, Wang S, Tang S, Liu C (2016) ABI 4 mediates antagonistic effects of abscisic acid and gibberellins at transcript and protein levels. The Plant Journal 85 (3):348-361

Son O, Hur YS, Kim YK, Lee HJ, Kim S, Kim MR, Nam KH, Lee MS, Kim BY, Park J, Park J, Lee SC, Hanada A, Yamaguchi S, Lee IJ, Kim SK, Yun DJ, Söderman E, Cheon CI (2010) ATHB12, an ABA-inducible homeodomain-leucine zipper (HD-Zip) protein of Arabidopsis, negatively regulates the growth of the inflorescence stem by decreasing the expression of a gibberellin 20-oxidase gene. Plant Cell Physiol 51(9):1537-47.

Sun TP (2008) Gibberellin metabolism, perception and signaling pathways in Arabidopsis. The Arabidopsis Book/American Society of Plant Biologists 6

Sun TP, Gubler F (2004) Molecular mechanism of gibberellin signaling in plants. Annu Rev Plant Biol 55:197-223

Sun TP (2010) Gibberellin-GID1-DELLA: a pivotal regulatory module for plant growth and development. Plant Physiol 154 (2):567-570. doi:10.1104/pp.110.161554

Takehara S, Sakuraba S, Mikami B, Yoshida H, Yoshimura H, Itoh A, Endo M, Watanabe N, Nagae T, Matsuoka M, Ueguchi-Tanaka M (2020) A common allosteric mechanism regulates homeostatic inactivation of auxin and gibberellin. Nat Commun 11 (1):2143. doi:10.1038/s41467-020-16068-0

Tan W, Han Q, Li Y, Yang F, Li J, Li P, Xu X, Lin H, Zhang D. (2021) A HAT1-DELLA signaling module regulates trichome initiation and leaf growth by achieving gibberellin homeostasis. New Phytologist 231(3):1220-1235.

Thomas SG, Blázquez MA, Alabadí D (2016) Della Proteins: Master Regulators of GibberellinResponsive Growth and Development. In: Annual Plant Reviews online. pp 189-227. doi:https://doi.org/10.1002/9781119312994.apr0536

Thomas SG, Phillips AL, Hedden P (1999) Molecular cloning and functional expression of gibberellin 2-oxidases, multifunctional enzymes involved in gibberellin deactivation. Proceedings of the National Academy of Sciences 96 (8):4698-4703

Urbanova T, Tarkowska D, Novak O, Hedden P, Strnad M (2013) Analysis of gibberellins as free acids by ultra performance liquid chromatography-tandem mass spectrometry. Talanta 112:85-94. doi:10.1016/j.talanta.2013.03.068

Wang H, Caruso LV, Downie AB, Perry SE (2004) The embryo MADS domain protein AGAMOUSLike 15 directly regulates expression of a gene encoding an enzyme involved in gibberellin metabolism. The Plant Cell 16 (5):1206-1219

Wang Y, Yu W, Ran L, Chen Z, Wang C, Dou Y, Qin Y, Suo Q, Li Y, Zeng J, Liang A, Dai Y, Wu Y, Ouyang X, Xiao Y (2021) DELLA-NAC interactions mediate GA signaling to promote secondary cell wall formation in cotton stem. Frontier in Plant Science 12:655127

Weller JL, Hecht V, Vander Schoor JK, Davidson SE, Ross JJ (2009) Light regulation of gibberellin biosynthesis in pea is mediated through the COP1/HY5 pathway. The Plant Cell 21 (3):800813

Willige BC, Ghosh S, Nill C, Zourelidou M, Dohmann EM, Maier A, Schwechheimer C (2007) The DELLA domain of GA INSENSITIVE mediates the interaction with the GA INSENSITIVE DWARF1A gibberellin receptor of Arabidopsis. The Plant Cell 19 (4):1209-1220

Wu A, Allu AD, Garapati P, Siddiqui H, Dortay H, Zanor M-I, Asensi-Fabado MA, Munné-Bosch S, Antonio C, Tohge T (2012) JUNGBRUNNEN1, a reactive oxygen species-responsive NAC transcription factor, regulates longevity in Arabidopsis. The Plant Cell 24 (2):482-506

Xue GP (2005) A CELD-fusion method for rapid determination of the DNA-binding sequence specificity of novel plant DNA-binding proteins. Plant Journal 41 (4):638-649. doi:10.1111/j.1365-313X.2004.02323.x

Yaish MW, El-Kereamy A, Zhu T, Beatty PH, Good AG, Bi YM, Rothstein SJ (2010) The APETALA-2-like transcription factor OsAP2-39 controls key interactions between abscisic acid and gibberellin in rice. PLoS Genet 6 (9):e1001098. doi:10.1371/journal.pgen.1001098 
Yamaguchi S, Kamiya Y (2000) Gibberellin biosynthesis: its regulation by endogenous and environmental signals. Plant and Cell Physiology 41 (3):251-257. doi:10.1093/pcp/41.3.251

Yamaguchi S, Kamiya Y, Sun Tp (2001) Distinct cell-specific expression patterns of early and late gibberellin biosynthetic genes during Arabidopsis seed germination. The Plant Journal 28 (4):443-453

Yamauchi Y, Takeda-Kamiya N, Hanada A, Ogawa M, Kuwahara A, Seo M, Kamiya Y, Yamaguchi S (2007) Contribution of gibberellin deactivation by AtGA2ox2 to the suppression of germination of dark-imbibed Arabidopsis thaliana seeds. Plant and Cell Physiology 48 (3):555-561

Yaxley JR, Ross JJ, Sherriff LJ, Reid JB (2001) Gibberellin biosynthesis mutations and root development in pea. Plant Physiology 125 (2):627-633

Yoo S-D, Cho Y-H, Sheen J (2007) Arabidopsis mesophyll protoplasts: a versatile cell system for transient gene expression analysis. Nature Protocols 2 (7):1565

Zaborowski AB, Walther D. (2020) Determinants of correlated expression of transcription factors and their target genes. Nucleic Acids Res 48(20):11347-11369

Zentella R, Zhang ZL, Park M, Thomas SG, Endo A, Murase K, Fleet CM, Jikumaru Y, Nambara E, Kamiya Y, Sun TP. (2007) Global analysis of DELLA direct targets in early gibberellin signaling in Arabidopsis. The Plant Cell 19(10):3037-3057.

Zhang S, Gottschalk C, van Nocker S (2019) Genetic mechanisms in the repression of flowering by gibberellins in apple (Malus x domestica Borkh.). BMC Genomics 20 (1):747

Zhou M, Chen H, Wei D, Ma H, Lin J (2017) Arabidopsis CBF3 and DELLAs positively regulate each other in response to low temperature. Sci Rep 7:39819. doi:10.1038/srep39819 


\section{Figure Legends}

Figure 1. Growth characteristics of $H B 40 O X$ and $h b 40-1$ mutant plants.

Plants were grown under long-day condition. (A) Phenotypes of wild-type (WT), $h b 40-1$, and $H B 40 O X$ plants, at 35 days after sowing (DAS). Scale bar, $5 \mathrm{~cm}$. (B) Quantification of the rosette area of plants shown in (A). Data represent means \pm s.d. $(n=16-23)$. (C) Typical leaf phenotypes. Fully expanded leaf no. 5 detached from 40-day-old WT, $h b 40-1$ and $H B 40 O X$ plants. Scale bar, $1 \mathrm{~cm}$. (D) Quantification of petiole length of plants shown in (C). (E) Quantification of leaf area of plants shown in (C). In (D) and (E), data represent means \pm s.d. (n = 6-9). (F) Flowering time and leaf number of WT, $h b 40-1$, and $H B 40 O X$ plants, defined as days from sowing to bolting (flower stem $\sim 0.5 \mathrm{~cm}$ ). Data represent means \pm s.d. $(\mathrm{n}=11-15)$. (G) HB40 transgenic plants compared to WT at 50 DAS. (H) Flowers of WT and HB40OX lines at floral stage13 (Cai and Lashbrook 2008). The arrows indicate shorter stamens in HB40OX compared to WT. Lower panel, quantification of stamen length $(\mathrm{n}=15)$. Asterisks denote significant differences relative to WT at $* * P<0.01$ by Student's $t$-test. (I) Hypocotyl length of seven-day-old dark-grown WT, HB40OX, and hb40-1 seedlings. Scale bar, $1 \mathrm{~cm}$. Lower panel, quantification of hypocotyl lengths. Data represent means \pm s.d. $(n=39-62)$. In (B), (D), (E), (F) and (I), asterisks denote significant differences relative to WT at $* P<0.05$, $* * P<0.01$ by Student's $t$-test.

\section{Figure 2. HB40 positively and directly regulates JUB1 expression.}

(A) Venn diagram of differentially expressed genes (fold change cut-off $\geq 2$ ) in estradiol ( $2 \mathrm{~h}$ ) vs. mock-treated HB4O-IOE seedlings, and HB4OOX vs. WT seedlings; age of seedlings was 10 days. Only genes whose promoters are targeted by HB40 in DNA affinity purification sequencing (DAP-seq) experiments and containing HD-Zip I binding sites are included. Upward arrows indicate upregulation, downward arrows indicate downregulation. (B) Expression of JUB1 measured by qRT-PCR in two-week-old WT, $h b 40-1$ and $H B 40 O X$ seedlings. The transcript level of HB40 in WT was set as 1. (C) Heat map showing transcript abundance of $H B 40$ and JUBI in 10-day-old HB40-HA-IOE seedlings after 2-8 $\mathrm{h}$ treatment with $10 \mu \mathrm{M}$ estradiol (Est) compared to the control (mock) treatment. The $\log 2$ fold change (FC) scale is indicated below the heat map. Data represent means of three biological replicates. (D) ChIP-qPCR demonstrates binding of HB40-HA to the JUB1 promoter. Ten-day-old HB4OHA-IOE seedlings were treated with $10 \mu \mathrm{M}$ Est for $1 \mathrm{~h}$ and harvested for ChIP. The Y-axis shows the fold enrichment of the ChIP DNA relative to the input. Gene TA3, which lacks an 
HB40 binding site, served as a negative control. Data represent means \pm s.d. (three independent biological replicates). Asterisks indicate significant difference in the enrichment of the promoter regions between estradiol- and mock-treated samples. ${ }^{*} P<0.05$, Student's $t$-test; NS, not significant. (E) ChIP-qPCR demonstrates binding of HB40-GFP to the JUB1 promoter. Ten-day-old HB40OX seedlings were analyzed. The Y-axis shows the fold enrichment of the ChIP DNA relative to the input. TA3, negative control. Data represent means \pm s.d. (three independent biological replicates). Asterisks indicate significant difference in the enrichment of the promoter regions between HB40-GFP and WT samples. ** $P<0.01$, Student's $t$-test; NS, not significant. (F) EMSA. Purified HB40-His protein binds to the HD-Zip I binding site within the JUB1 promoter. Lane 1, labeled probe (5'-DY682-labeled double-stranded oligonucleotide); lane 2, labeled probe plus HB40-His protein; lane 3, labelled probe, HB40His protein, and unlabeled competitor (oligonucleotide containing HB40 binding site; $200 \mathrm{x}$ molar access); lane 4, labeled probe, HB40-His protein, and unlabeled mutated competitor (oligonucleotide containing mutated HB40 binding site; 200 x molar access). The arrow indicates the retarded band. (G) Transactivation of $J U B 1$ expression (from its 1-kb promoter) by HB40 in Arabidopsis mesophyll cell protoplasts. The JUB1:LUC construct harboring the JUB1 promoter upstream of the firefly (Photinus pyralis) luciferase open reading frame was transformed with or without the $35 \mathrm{~S}: \mathrm{HB} 40$ plasmid into the protoplasts. Data represent means \pm s.d. $(\mathrm{n}=3)$. (H) Activation of the JUB1 promoter by $\mathrm{HB} 40$ in the $\mathrm{Y} 1 \mathrm{H}$ assay. A 373 bp JUB1 promoter containing the HD-Zip I binding site was used. HB40 was fused to the GAL4 activation domain (HB40-AD) in pDEST22. Upon interaction of HB40-AD with its binding site within the $J U B 1$ promoter, transcription of the yeast $H I S 3$ reporter gene is activated and diploid yeast cells grow on selective medium -Leu/-Trp/-His (-L-T-H) with 3-amino-1, 2,4triazole (3-AT). The empty vector (EV, pDEST22) with AD alone was used as a negative control. (I) Hypocotyl lengths of six-day-old dark-grown HB40OX/jub1-1 seedlings alongside WT, HB40OX, and jubl-1 seedlings. Scale bar, $1 \mathrm{~cm}$. Lower panel, Quantification of lengths of hypocotyls shown in (I). Data represent means \pm s.d. $(\mathrm{n}=24-49)$. (J) HB40OX plants compared to WT, HB4OOX/jubl-1 and jubl-1 at three weeks after sowing. Plants were grown under long-day condition. Scale bar, $2 \mathrm{~cm}$. Lower panel, quantification of the rosette area of plants shown in $(\mathrm{J})$. Data represent means \pm s.d. $(\mathrm{n}=7-14)$. (K) Mature HB40OX, JUB1OX, WT, and HB40OX/jub1-1 plants at 34 DAS (days after sowing) grown under long-day conditions. (L) Flowering time (DAS) of WT, HB40OX, HB40OX/jub1-1 and jub1-1 plants grown under long-day condition, defined as days from sowing to bolting (flower stem $\sim 0.5 \mathrm{~cm}$ ). Data represent means \pm s.d. $(n=11-13)$. In $(B),(C)$, and $(G)$, significant differences from 
corresponding controls are indicated; $* P<0.05, * * P<0.01$, Student's $t$-test. In (I), (J) and (L), letters indicate significant differences between means $(P<0.05$; one-way ANOVA).

\section{Figure 3. HB40 reduces GA biosynthesis and enhances GA inactivation.}

(A) Hypocotyl lengths of seven-day-old WT and HB40OX seedlings grown on half-strength MS medium under light condition supplemented with or without $200 \mathrm{nM} \mathrm{GA} 4$ and/or $100 \mathrm{nM}$ BL. Data represent means \pm s.d. $(\mathrm{n}=25-42)$. (B) Five-day-old WT, $h b 40-1$, and HB40OX seedlings were transferred to $\mathrm{GA}_{4}$-containing or mock medium and grown under short-day conditions. Photographs were taken 32 days later. Scale bar, $2 \mathrm{~cm}$. (C) Quantification of stem lengths of data shown in (B). Data represent means \pm s.d. $(n=10-11)$. ND, no flower bolts detected. In (A) and (C), letters indicate significant differences between means $(P<0.05$; oneway ANOVA). (D) and (E) Concentration of bioactive GAs, $\mathrm{GA}_{1}$ and $\mathrm{GA}_{4}$, in 10-day-old seedlings of WT, hb40-1, HB4OX and HB40OX/jubl-1\#1 (D), and 10-day-old HB4O-HA-IOE seedlings after $8 \mathrm{~h}$ treatment with $10 \mu \mathrm{M}$ Est (E). (F) and (G) Concentration of bio-inactive GAs, GA51, GA $29, \mathrm{GA}_{34}$ and $\mathrm{GA}_{8}$, in 10-day-old seedlings of WT, hb40-1, HB4OX and HB40OX/jubl-1 (F), and 10-day-old HB40-HA-IOE seedlings after $8 \mathrm{~h}$ treatment with $10 \mu \mathrm{M}$ Est (G). In (D) - (G), data represent means (pg/mg fresh weight) \pm s.d. (three biological replicates). Asterisks indicate significant differences from WT or mock treatments; ${ }^{*} P<0.05$, $* * P<0.01$, Student's $t$-test.

\section{Figure 4. HB40 directly regulates GA catabolism genes.}

(A) Heat map showing transcript abundance of GA catabolism genes including GA2oxl, GA2ox2, GA2ox3, GA2ox4 and GA2ox6 in 10-day-old HB40-HA-IOE seedlings after $8 \mathrm{~h}$ treatment with $10 \mu \mathrm{M}$ estradiol (Est) compared to the control (mock) treatment. The log2 fold change scale is indicated below the heat map. Data represent means of three biological replicates. Asterisks denote significant differences relative to mock; $* P<0.05, * * P<0.01$, Student's $t$-test. (B) Expression of GA2ox2 and GA2ox6 measured by qRT-PCR in four-dayold WT and HB40OX seedlings. (C) Expression of GA2ox2 and GA2ox6 measured by qRTPCR in 15-day-old WT and $h b 40-1$ shoots. In (B) and (C), data represent means \pm s.d. (three biological replicates) and asterisks denote significant difference from WT; ${ }^{*} P<0.05$, $* * P<$ 0.01 , Student's $t$-test. (D) Schemes of GA2ox2 and GA2ox6 promoters showing HB40 binding sites located at $1,010 \mathrm{bp}$ and $643 \mathrm{bp}$ upstream of the translation start codon (ATG) in the respective promoters. (E) EMSA. Purified HB40-His protein binds to HD-Zip I binding sites within the GA2ox2 (left) and GA2ox6 (right) promoters. From left to right in each image: lane 
1, labeled probe (5'-DY682-labelled double-stranded oligonucleotides); lane 2, labeled probe plus HB40-His protein; lane 3, labelled probe, HB40-His protein, and competitor (unlabeled oligonucleotide containing HB40 binding site; 200 x molar access). Arrows indicate retarded bands ('Bound oligo') and the non-bound DNA probes ('Free oligo'). (F) ChIP-qPCR demonstrates binding of HB40-GFP to GA2ox2 and GA2ox6 promoters. Ten-day-old seedlings of HB40OX (harboring HB40 in fusion with GFP) were used for the assay. (G) ChIP-qPCR demonstrates binding of HB40-HA to GA2ox2 and GA2ox6 promoters. Ten-day-old HB40-IOE seedlings (harboring HB40 in fusion with HA) were treated with $10 \mu \mathrm{M}$ Est for $1 \mathrm{~h}$ and harvested for ChIP. In (F) and (G), the Y-axis shows the fold enrichment of the ChIP DNA relative to the input. Gene TA3, which lacks an HB40 binding site, served as a negative control. Data represent means \pm s.d. (three independent biological replicates). Asterisks indicate significant difference in the enrichment of the promoter regions between HB40-GFP and WT (F) or estradiol- and mock-treated $(\mathrm{G})$ samples. ${ }^{*} P<0.05$, ** $P<0.01$, Student's $t$-test; NS, not significant. (H) Binding of HB40 to the promoter of GA2ox6 in the Y1H assay. A 1000bp GA2ox6 promoter containing the wild-type or mutated HD-Zip I binding sites were used. HB40 was fused to the GAL4 activation domain (HB40-AD) in pDEST22. Upon interaction of HB40-AD with its binding site within the GA2ox6 promoter, transcription of the yeast HIS3 reporter gene is activated and diploid yeast cells grow on selective medium -Leu/Trp/-His (-L-T-H) with 3-amino-1,2,4-triazole (3-AT) at $10 \mathrm{mM}$ and $20 \mathrm{mM}$. The empty vector (EV) served as a negative control.

Figure 5. Growth and developmental defects of $\mathrm{HB} 40 \mathrm{OX}$ are largely recovered by GA2ox mutations and dimethyl GA4 treatment.

(A) Hypocotyl lengths of seven-day-old dark-grown HB40OX/ga2oxs lines alongside WT, HB40OX, and ga2oxs. Data represent means \pm s.d. (n = 15-38). (B) Phenotype of WT, HB40OX, HB40OX/ga2oxs and ga2oxs plants at 30 days after sowing (DAS). Scale bar, $5 \mathrm{~cm}$. (C) Quantification of rosette area of plants shown in (B) Data represent means \pm s.d. $(n=7)$. (D) Flowering time and number of leaves of WT, HB40OX, ga2oxs and HB40OX/ga2oxs plants grown under long-day conditions, at 48 DAS. Data represent means \pm s.d. $(\mathrm{n}=9)$. (E) Phenotype of WT, HB40OX, HB40OX/ga2oxs and ga2oxs plants at 54 days after sowing (DAS). Scale bar, $5 \mathrm{~cm}$. (F) Quantification of height of plants shown in (E). Data represent means \pm s.d. ( $\mathrm{n}=9)$. In (A), (C), (D) and (F), asterisks denote significant differences from WT; $* P<0.05$, $* * P<0.01$, Student's $t$-test. NS, not significant. (G) Hypocotyls of seven-day-old WT and $H B 40 O X$ seedlings grown on half-strength MS medium under dark conditions in the 
absence or presence of $100 \mathrm{nM} \mathrm{GA} 4$ or $100 \mathrm{nM}$ 2,2-dimethyl GA4. Scale bar, $0.5 \mathrm{~cm}$. (H) Quantification of hypocotyl lengths. Data represent means \pm s.d. $(\mathrm{n}=16-34)$. (I) Five-day-old WT, $H B 40 O X$ and $h b 40-1$ seedlings were transferred to $0.5 \mu \mathrm{M} \mathrm{GA}_{4}$ - or 2,2-dimethyl $\mathrm{GA}_{4}$ containing medium and photographs were taken two weeks later. Scale bar, $1 \mathrm{~cm}$. Inset, a closer look at the $H B 40 O X$ plants upon treatment with 2,2-dimethl GA 4 . (J) Primary inflorescence lengths of plants shown in (I). Data represent means \pm s.d. $(n=9-15)$. In $(H)$ and (J), letters indicate significant differences between means $(P<0.05$; one-way ANOVA). ND, not detected.

\section{Figure 6. HB40 inhibits growth and development via DELLAs.}

(A) Western blot analysis of RGA protein (by anti-RGA antibodies) in seven-day-old darkgrown seedlings of WT, HB40OX, hb40-1 (left panel), and 10-day-old HB4O-HA-IOE seedlings after $8 \mathrm{~h}$ estradiol (Est, $10 \mu \mathrm{M}$ ) or mock (control) treatment (right panel). RbcL, ribulose-1,5bisphosphate carboxylase/oxygenase large subunit (loading control; Ponceau S staining). kDa, kilodalton. Signals of immunoblot analyses were quantified by ImageJ (https://imagej.net/Fiji). Relative intensities (RGA : RbcL) are shown as numerical values. Data represent means (three biological replicates). Asterisks denote significant differences from WT at $* P<0.05$, Student's t-test. (B) HB40OX/Ler plants compared to Ler, penta della and HB40OX/penta della at three weeks after sowing (DAS). Plants were grown under long-day condition. Scale bar, $2 \mathrm{~cm}$. (C) Quantification of the rosette area of plants shown in (B). Data represent means \pm s.d. $(n=7$ 13). (D) Mature Ler, HB40OX/Ler, HB40OX/penta della and penta dellla plants grown under long-day condition at 35 DAS. Scale bar, $5 \mathrm{~cm}$. (E) Flowering time of HB40OX/Ler, HB40OX/penta della, penta dellla and Ler plants grown under long-day condition. Data represent means \pm s.d. $(\mathrm{n}=7-13)$. In $(\mathrm{C})$ and $(\mathrm{E})$, asterisks denote significant differences from WT; **P $<0.01$, Student's $t$-test. (F) Mature Ler, HB40OX/Ler, HB40OX/penta della and penta dellla plants grown under long-day condition, at 50 DAS. Scale bar, $5 \mathrm{~cm}$. (G) Quantification of the height of plants shown in $(F)$. Data represent means \pm s.d. $(n=22-26)$.

(H) Hypocotyls of 7-day-old Ler, HB40OX/Ler, HB40OX/penta della and penta della seedlings grown in darkness on half-strength MS agar plates. Scale bar, $1 \mathrm{~cm}$. (I) Quantification of hypocotyl lengths. Data represent means \pm s.d. $(n=53-72)$. In $(G)$ and $(I)$, asterisks denote significant differences relative to Ler at $* P<0.05$, $* * P<0.01$, Student's $t$-test. (J) Flowers of Ler, penta della, HB40OX/Ler and HB40OX/penta della plants at floral stage 13 (Cai and Lashbrook 2008). The arrow indicates shorter stamens in HB40OX/Ler. Scale bar, $1 \mathrm{~mm}$. 
Figure 7. A model for the action of $\mathrm{HB} 40$ in the regulation of GA-mediated growth in Arabidopsis.

HB40 negatively regulates the levels of bioactive GAs, in part by directly activating the JUB1 transcription factor and thereby suppressing GA3ox expression resulting in less active GAs and a higher accumulation of GA signaling repressors, DELLA proteins. In addition, HB40 promotes the accumulation of bio-inactive GAs and reduces bioactive GAs by directly upregulating the expression of GA-catabolic enzymes, GA2oxs. Lower levels of bioactive GAs lead to increased DELLA protein levels, thereby suppressing various growth and developmental processes. HB40 expression is induced by GA in a DELLA-dependent manner, indicating that regulation of GA homeostasis is composed of an autoregulatory negative feedback loop formed by GA, DELLAs and HB40. The figure was prepared using BioRender (www.biorender.com). 
A

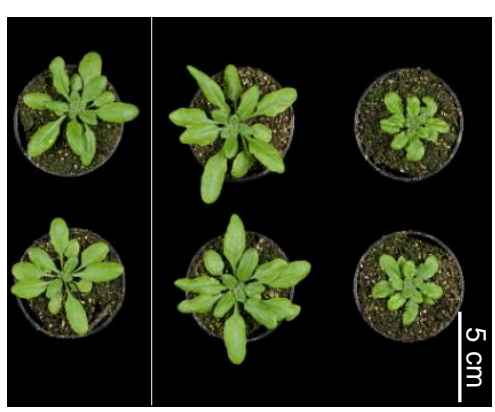

WT hb40-1 HB40OX
B

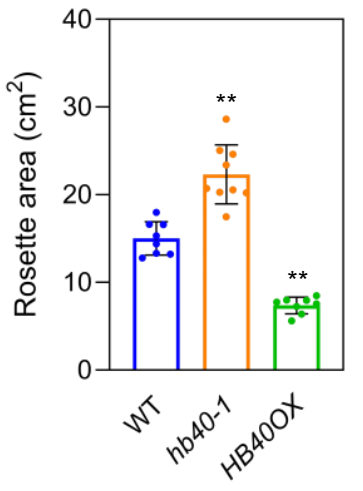

C

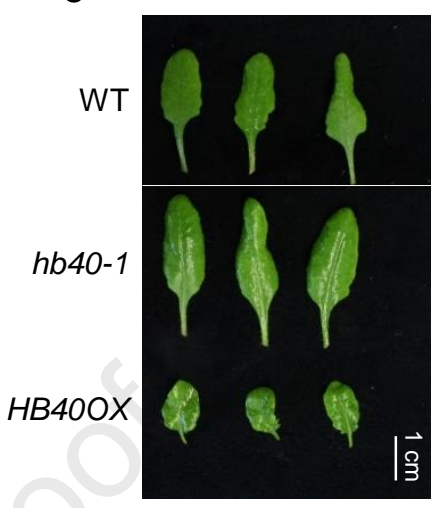

D

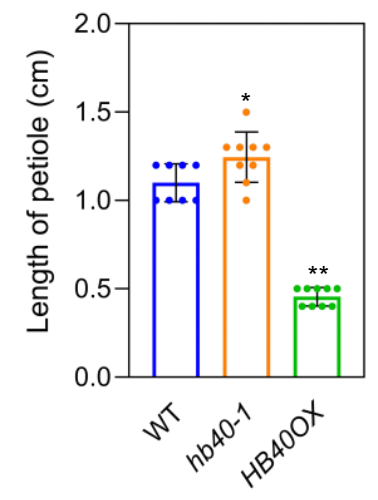

G

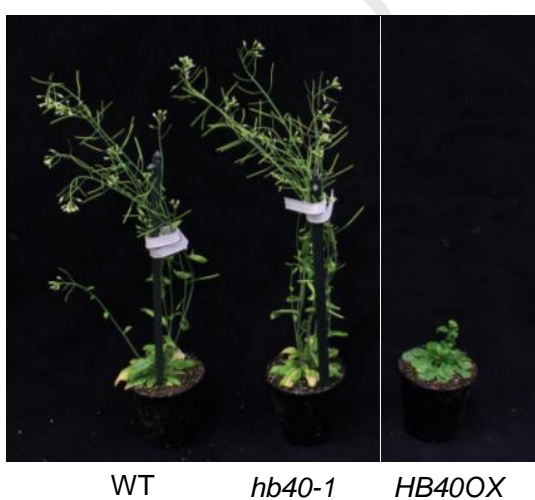

E

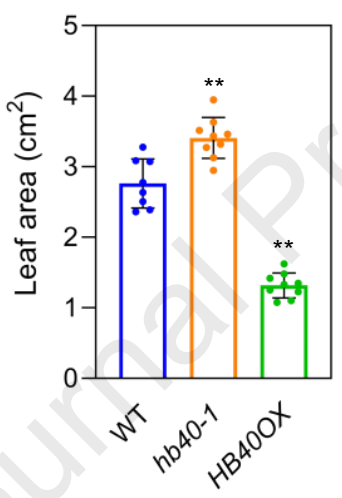

$\mathbf{F}$

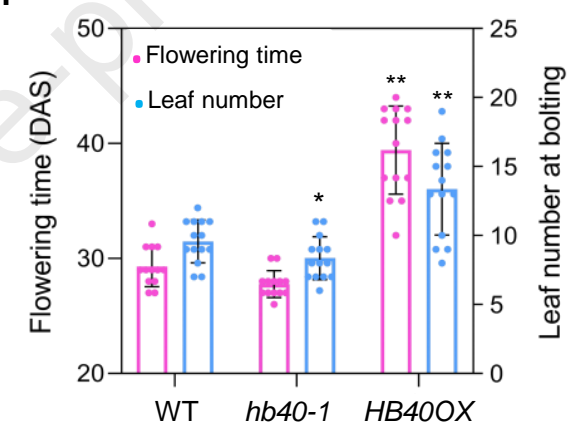

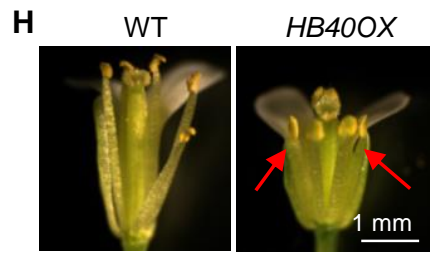

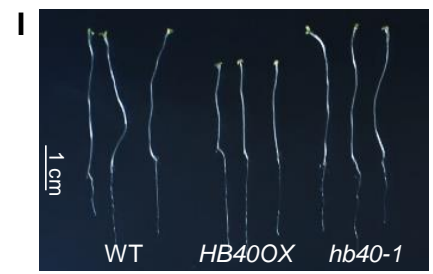

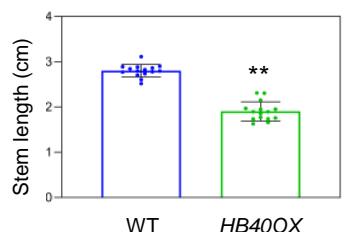

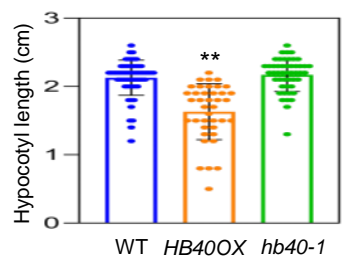


A

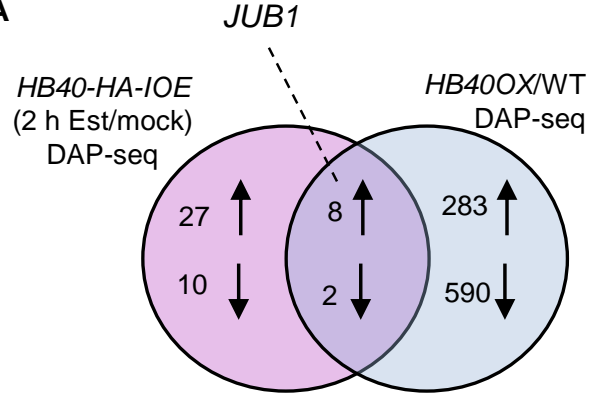

B

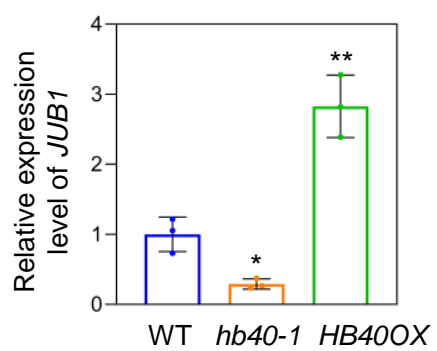

C

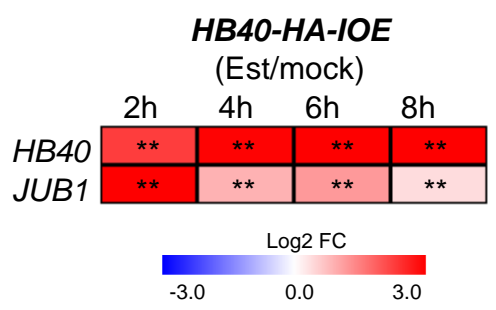

D

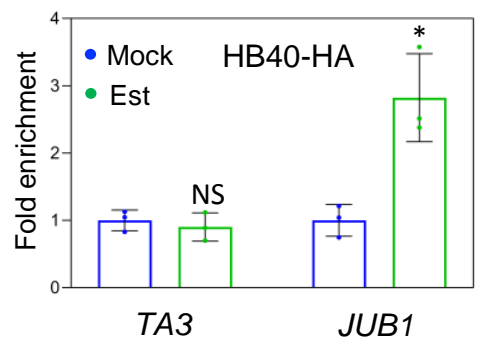

H
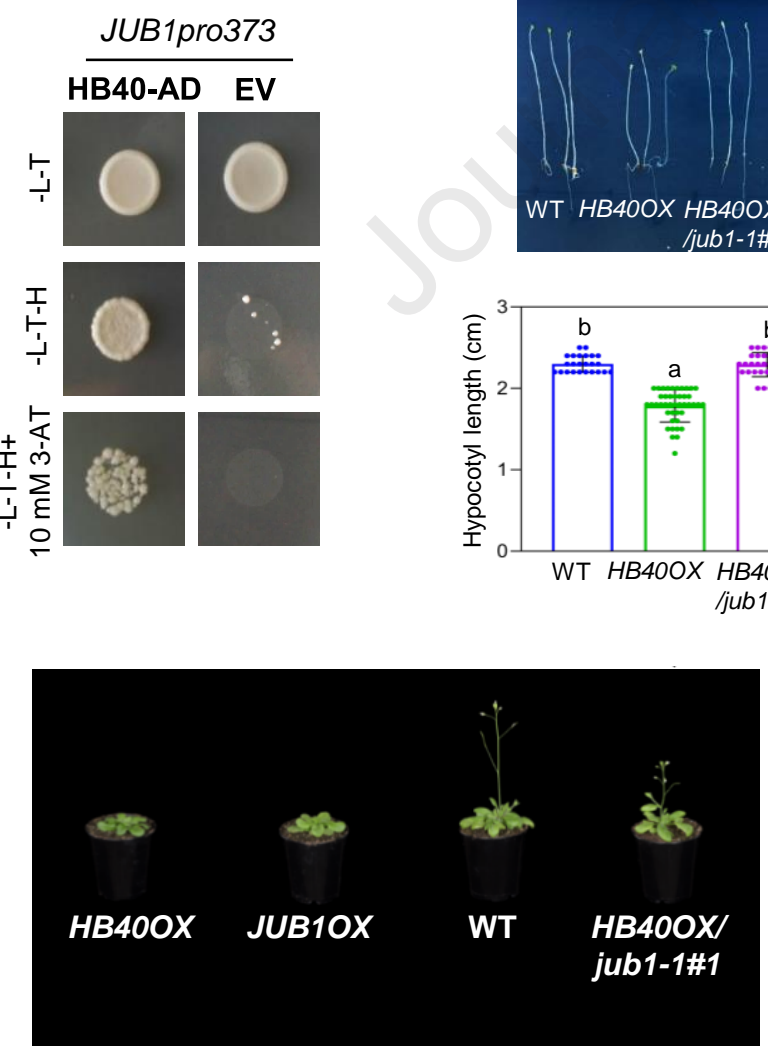

E

I
K

jub1-1\#1
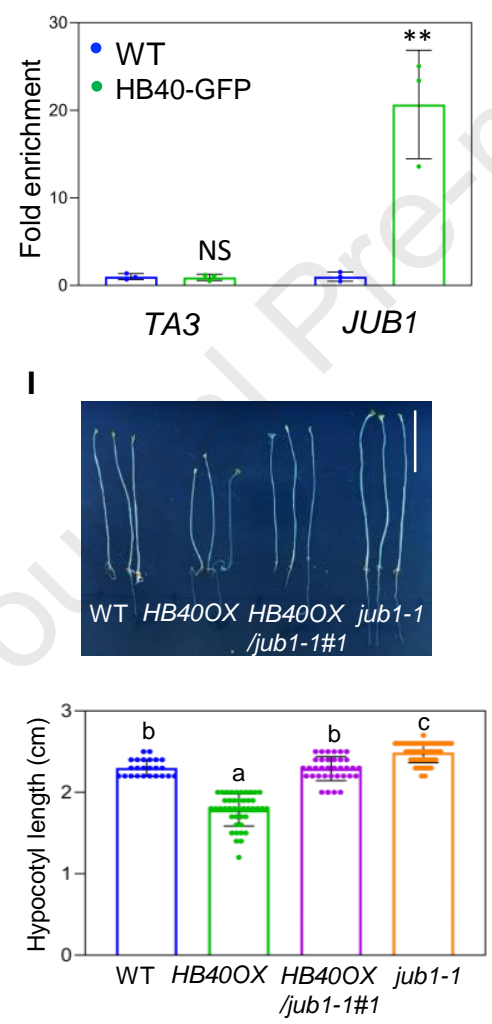

L

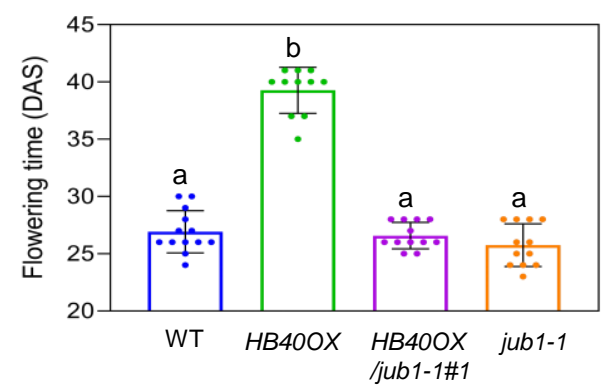

F

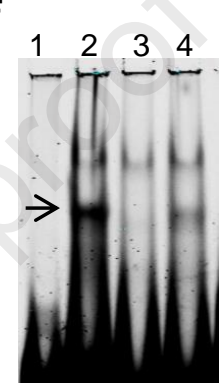

G

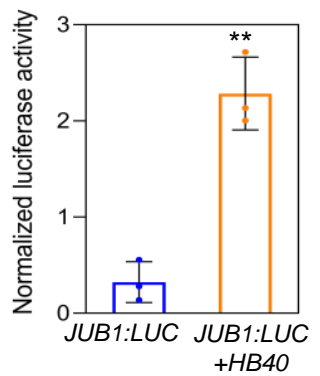

J
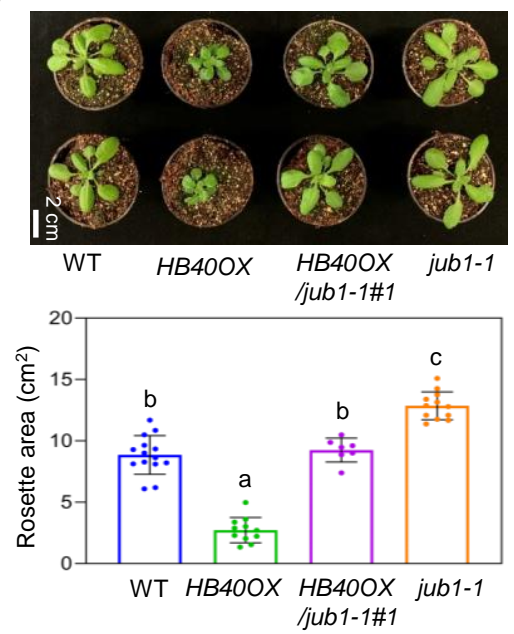

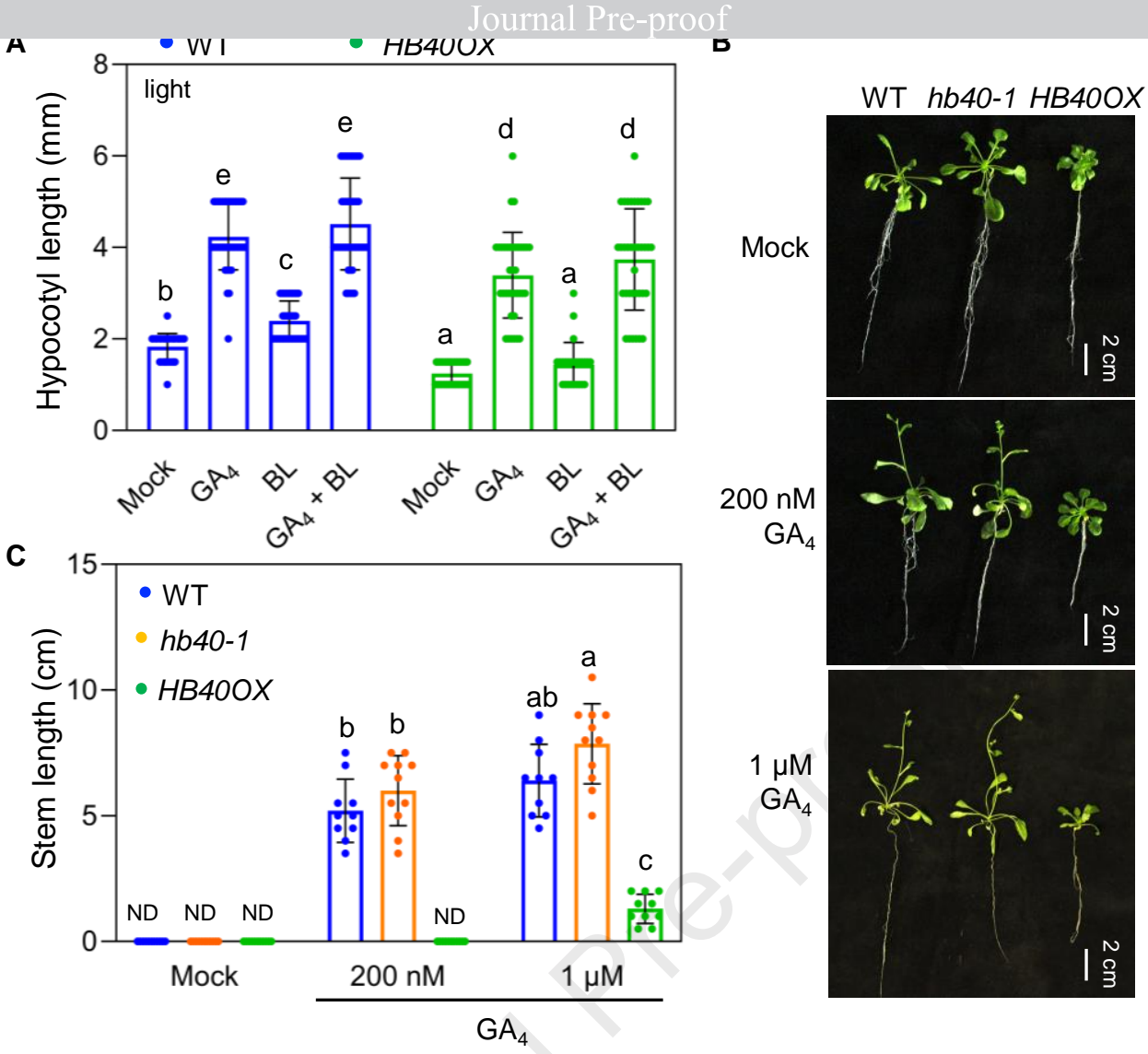

D

$$
\text { - WT - HB40OX }
$$

- hb40-1 HB40OXIjub1-1
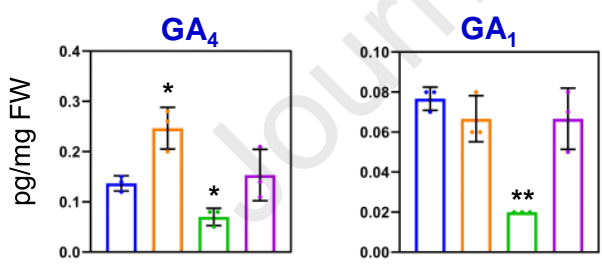

F
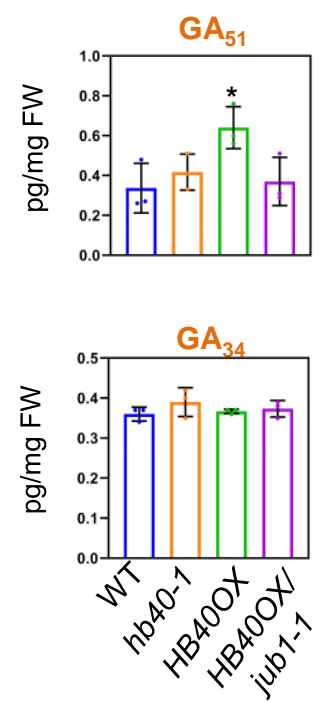

D

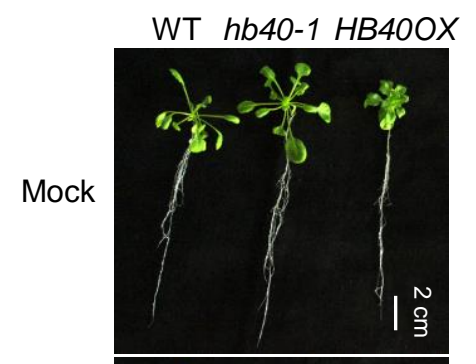

$1 \mu \mathrm{M}$

E
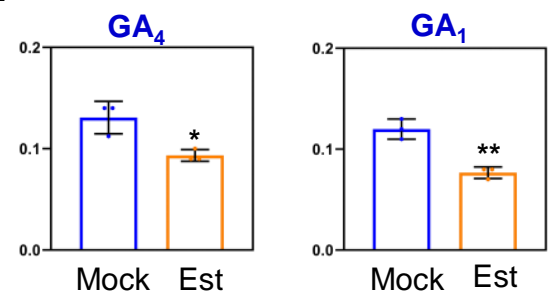

G
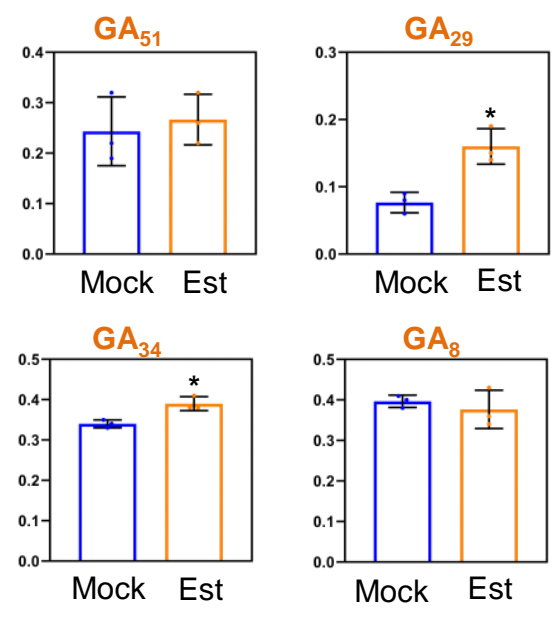
A

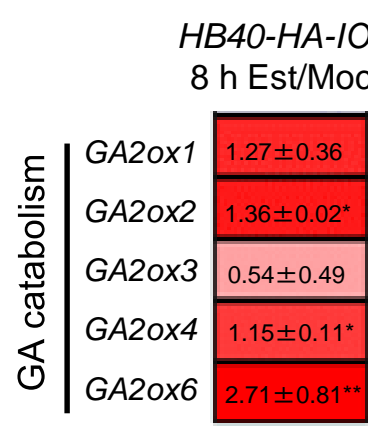

Log2 FC

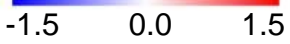

D

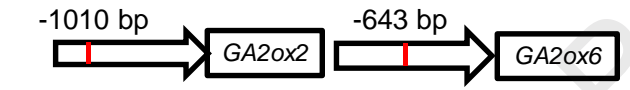

E

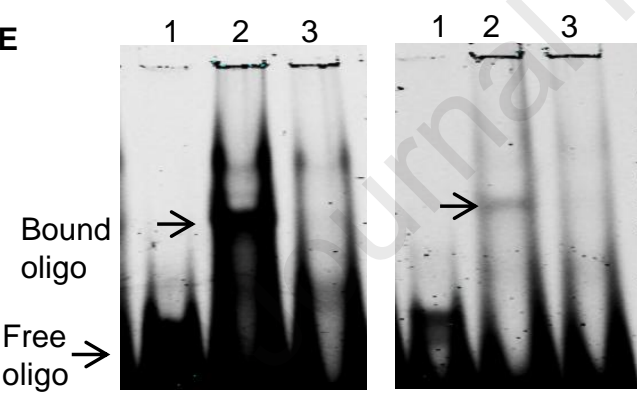

G

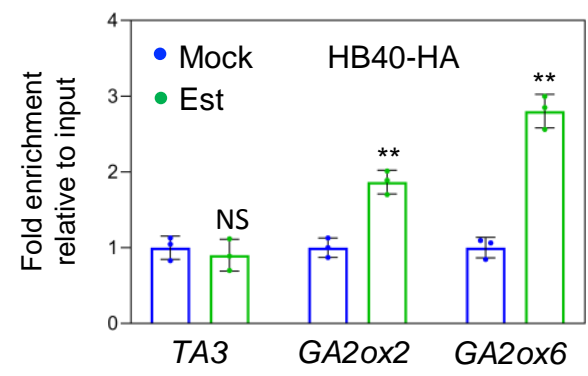

H
B
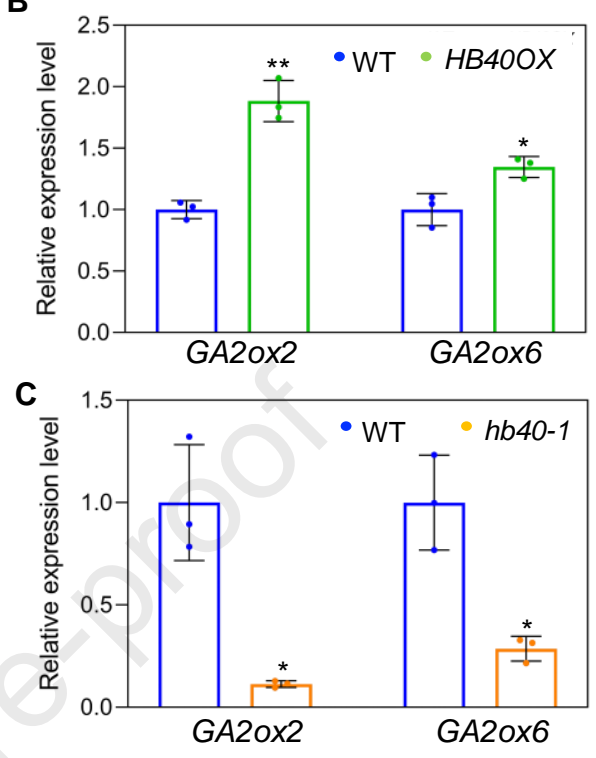

F

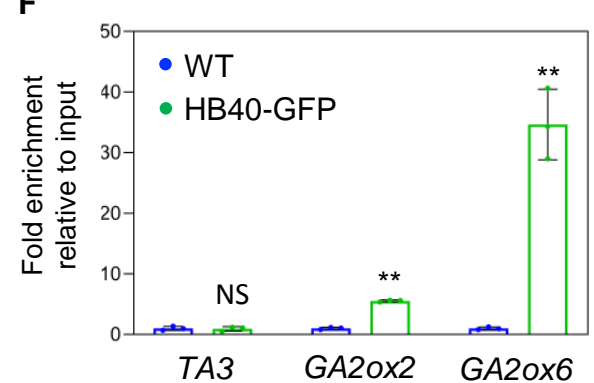

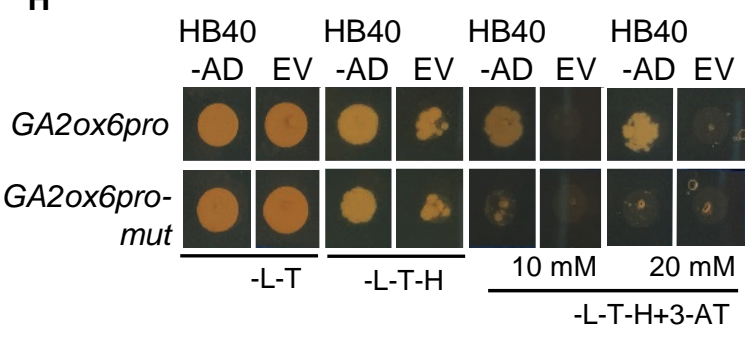




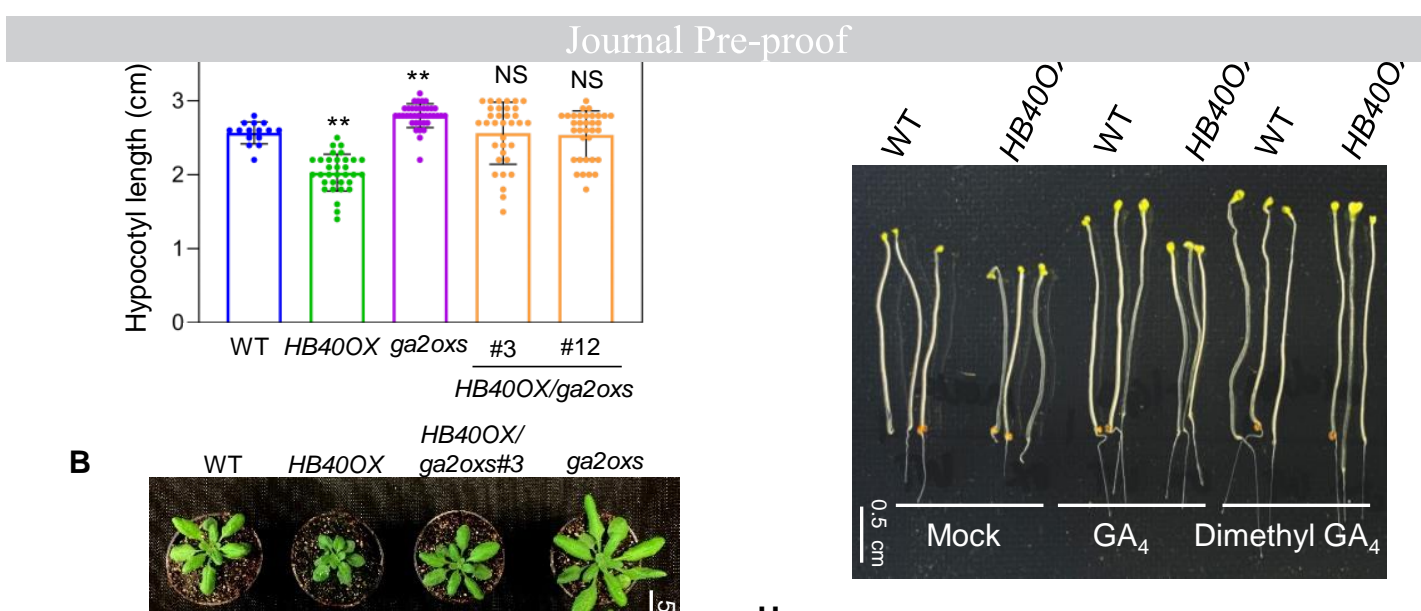

H

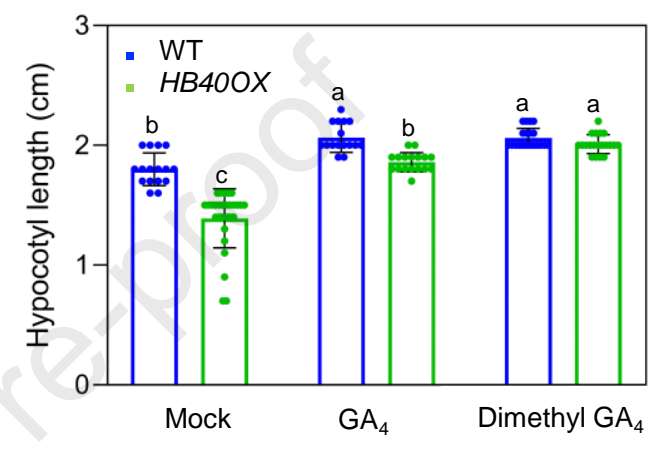

D
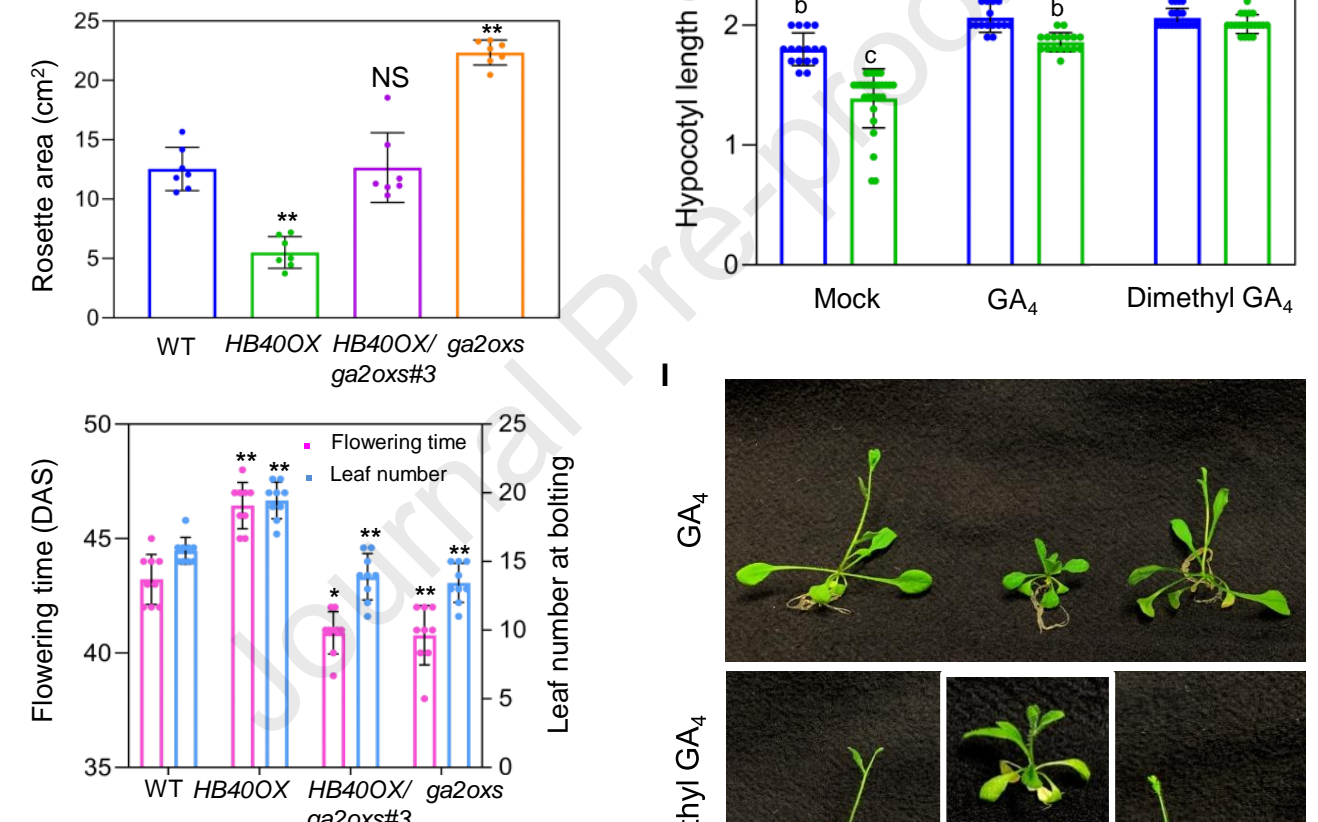

E

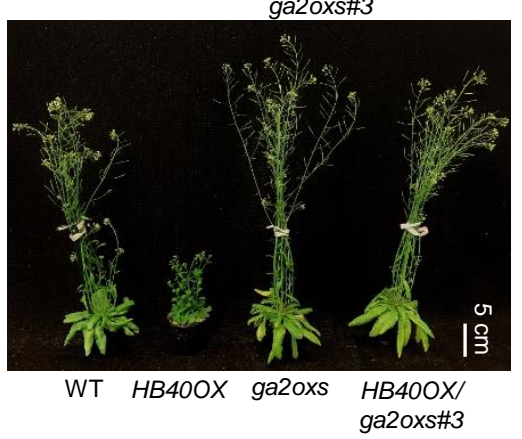

F
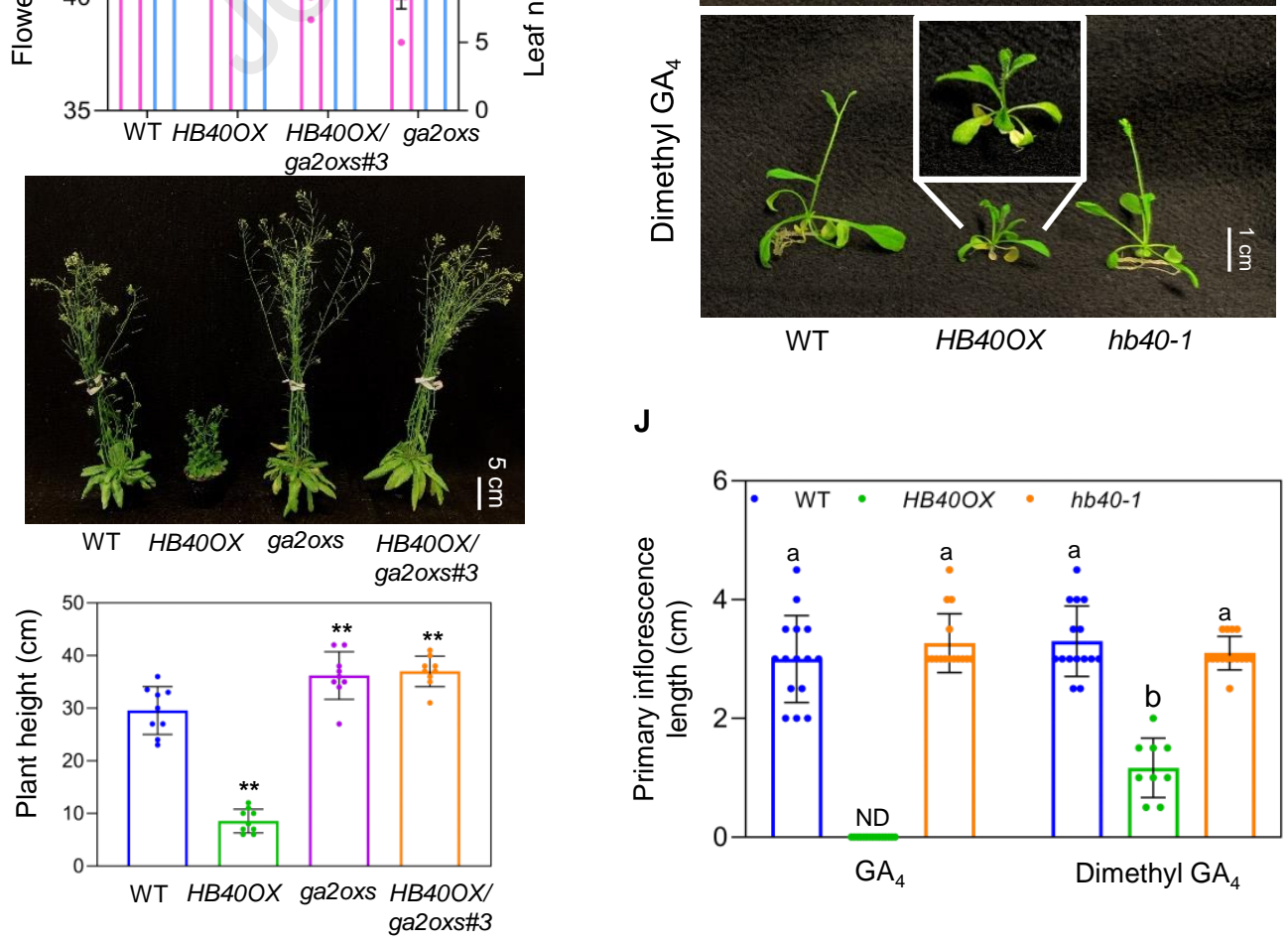

J

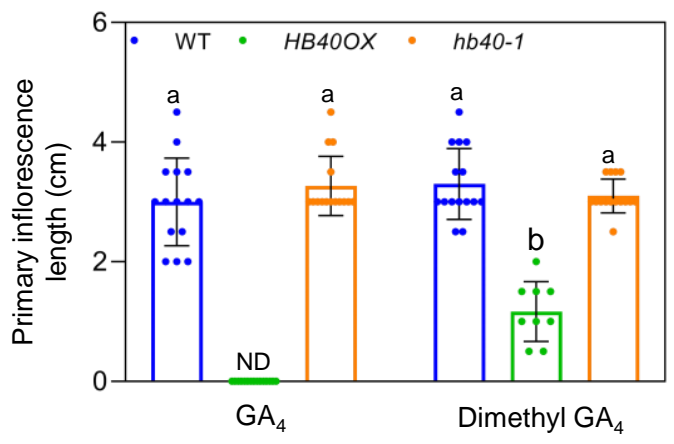




\section{Figure 6}

A

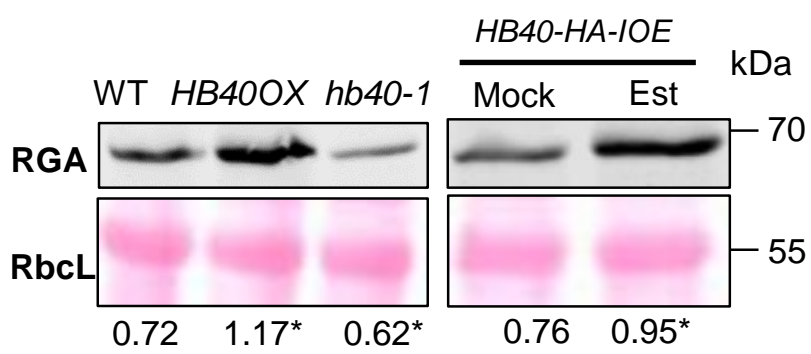

B

HB40OX/ HB40OX/

Ler pentadella penta

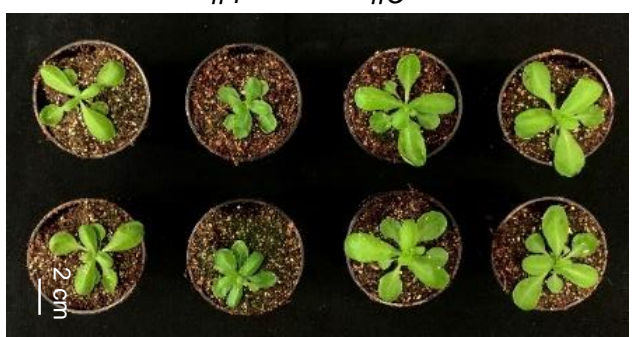

C

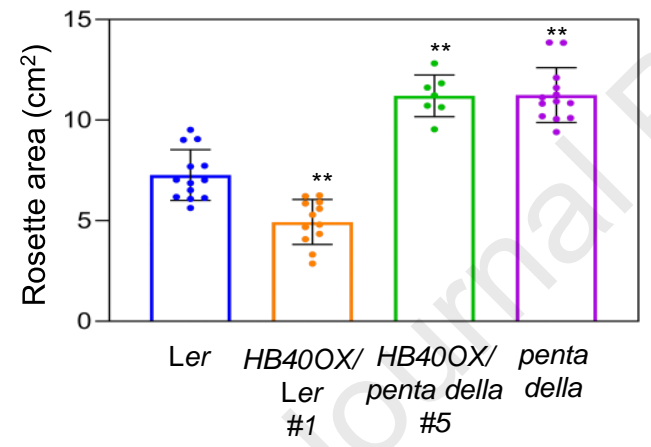

D

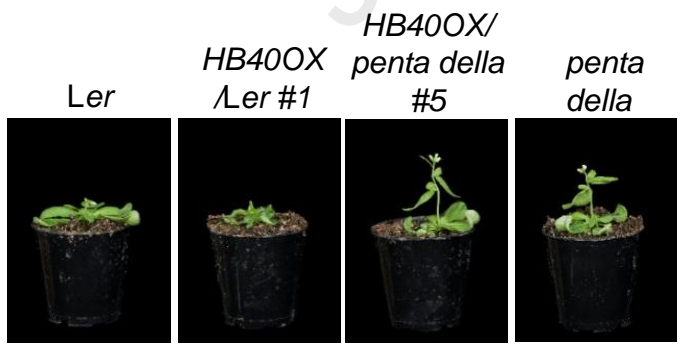

E

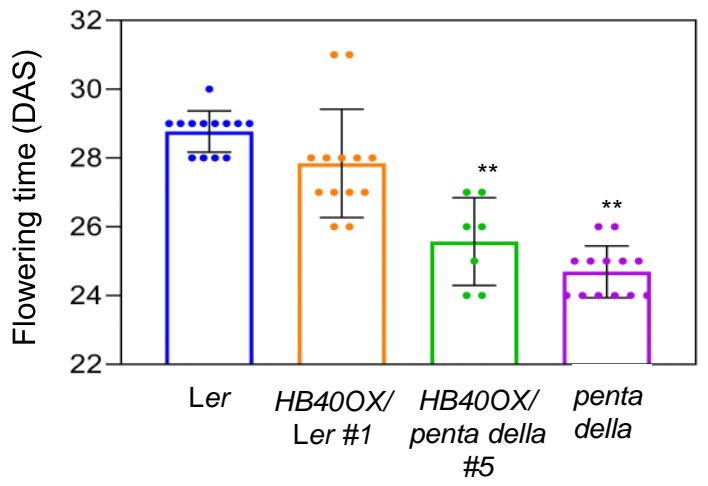

$\mathbf{F}$

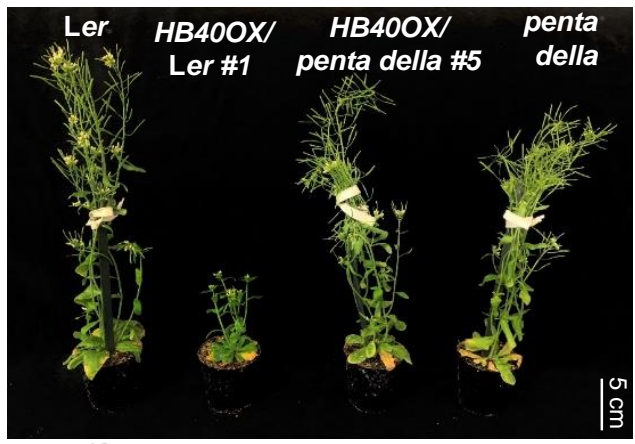

G

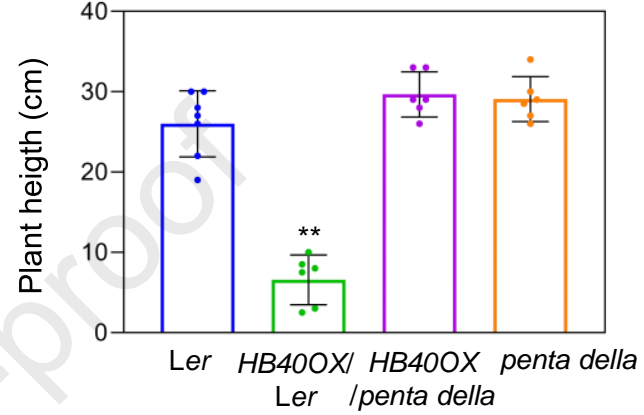

H

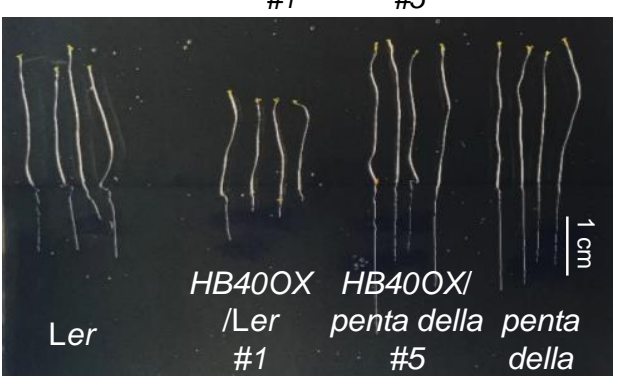

I

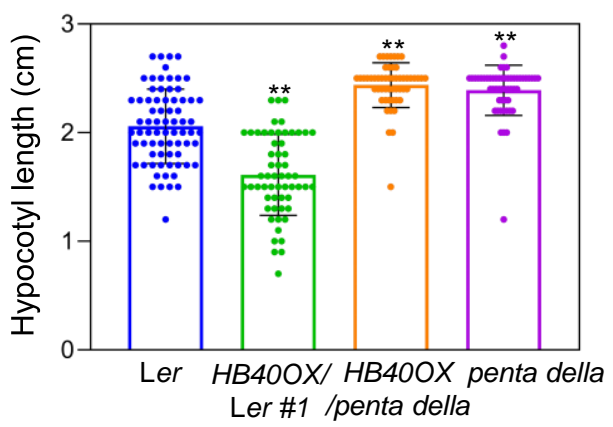

J \#5

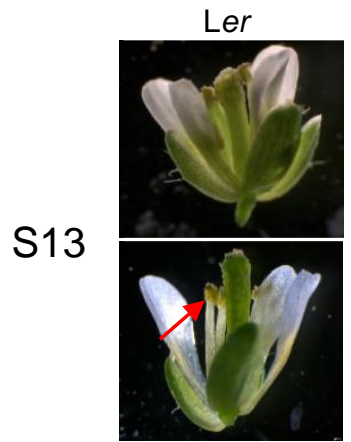

HB40OXI

Ler \#1 penta della

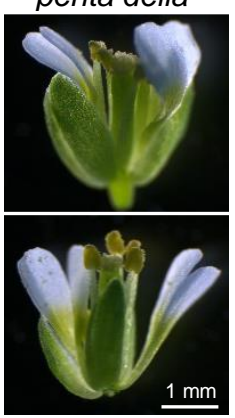

HB40OXI

penta della \#5 
Figure 7

HB40

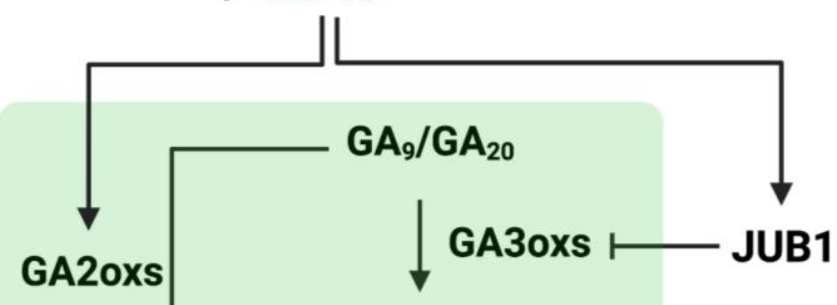

$\mathbf{G A}_{4} / \mathbf{G A}_{1}$

Bioactive GAs

Inactive

GAs

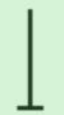

DELLAs

GA response

(elongation growth) 